\title{
Wake behind a three-dimensional dry transom stern. Part 1: Flow structure and large-scale air entrainment
}

\author{
Kelli Hendrickson $†$, Gabriel D. Weymouth ${ }^{1}$, Xiangming Yu, and Dick \\ K.-P. Yue \\ Department of Mechanical Engineering, Massachusetts Institute of Technology, Cambridge, \\ MA 02139, USA \\ ${ }^{1}$ Present address: Southampton Marine and Maritime Institute, University of Southampton, \\ SO16 7QF, UK
}

(Received $\mathrm{xx}$; revised $\mathrm{xx}$; accepted $\mathrm{xx}$ )

We present high-resolution implicit large eddy simulation (iLES) of the turbulent air entraining flow in the wake of three-dimensional rectangular dry transom sterns with varying speeds and half-beam-to-draft ratios $B / D$. We employ two-phase (air/water), time-dependent simulations utilizing conservative Volume-of-Fluid (cVOF) and Boundary Data Immersion (BDIM) methods to obtain the flow structure and large-scale air entrainment in the wake. We confirm that the convergent corner wave region that forms immediately aft of the stern wake is ballistic, thus predictable only by the speed and (rectangular) geometry of the ship. We show that the flow structure in the air-water mixed region contains a shear layer with a streamwise jet and secondary vortex structures due to the presence of the quasi-steady, three-dimensional breaking waves. We apply a Lagrangian cavity identification technique to quantify the air entrainment in the wake and show that the strongest entrainment is where wave breaking occurs. We identify an inverse dependence of the maximum average void fraction and total volume entrained with $B / D$. We determine that the average surface entrainment rate initially peaks at a location that scales with draft-Froude number and that the normalized average air cavity density spectrum has a consistent value providing there is active air entrainment. A small parametric study of the rectangular geometry and stern speed establishes and confirms the scaling of the interface characteristics with draft-Froude number and geometry. In part 2 we examine the incompressible highly-variable density turbulence characteristics and turbulence closure modeling.

Key words:

\section{Introduction}

Our interest is the complex, three-dimensional wake immediately behind the dry transom stern of a surface ship. This region contains violent breaking of the free surface, highly mixed air-water turbulent flow, large-scale air entrainment, and air cavity breakup. In addition to gaining fundamental scientific understanding, quantification and prediction of these features are of critical importance to the design and operation of surface ships. The objective of the present work is to perform iLES to elucidate and quantify

$\dagger$ Email address for correspondence: khendrk@mit.edu 
the near-wake of a dry transom, to complement and support the laboratory and field measurements, and to inform bubble source and turbulence closure models used in largescale (whole-ship) predictions (Jingsen et al. 2011; Ma et al. 2011; Baldy 1993). Presented in two parts, part 1 focuses on the wake surface, the structure of the underlying wake flow, Lagrangian characteristics of the large-scale air entrainment and surface entrainment rate, and the scaling of these with Froude number and stern geometry. The objective of this part is to provide a physical understanding of the detailed flow features in the mixed region and the characteristics of the large-scale air entrainment. Part 2 (Hendrickson \& Yue submitted) focuses on understanding and modeling of the highly-mixed, air-water turbulent near-surface region of the wake by combining the the surface fluctuations, spray and entrainment into a single mixed-phase region through an Eulerian framework. The objective of this part is to provide a statistical understanding of the mixed-phase region within the context of incompressible highly variable density turbulence and address the necessary turbulent mass flux closure modeling.

While there are many studies of small-scale air entrainment such as for a surface impinging jet (Zhu et al. 2000; Ohl et al. 2000; Cummings \& Chanson 1997; Chanson \& Manasseh 2003; Chanson et al. 2004; Hoque \& Aoki 2008), the ship-wake problem presents special experimental and computational challenges. In addition to the complexities of the air-water interface and entraining turbulent flows, the (prototype) problem involves high void fractions (20-30\%) (Terrill \& Fu 2008) and flow length scales spanning from the ship length, beam and draft $O\left(10^{1-2}\right) \mathrm{m}$ down to micron-sized bubbles. Indeed, until recently, relatively little is understood or predictable about this flow.

Recent full-scale experiments of a transom stern vessel (Terrill \& Fu 2008; Terrill \& Taylor 2015; Fu et al. 2006) and laboratory-scale experiments of a transom hull form (Drazen et al. 2010) and breaking waves (Lamarre \& Melville 1991) provided significant insight into wake interface characteristics and statistics as well as initial information regarding the wake air entrainment. Based on images from experiments ( $\mathrm{Fu}$ et al. 2010a), the three-dimensional flow behind the transom stern is visually similar to the supercritical flow behind chute piers in spillways due to the presence of the characteristic rooster tail (Pagliara et al. 2011). Laboratory scale experiments reported void fractions within 10$15 \%$ and field scale experiments at 20-30\%. Experiments at both scales reported the location of the bulk of the entrained air to be immediately aft of the stern in the rooster tail region and confined within the depth of the wetted transom. Due to the complexity of full-scale at-sea measurements and measurements with high void fractions, there is currently no published data on the detailed flow structure or air entrainment in the mixed region of the wake of a ship at either scale.

State-of-the-art multiphase viscous flow simulations have advanced numerical capabilities for modeling the complex gas-liquid flows of hydraulic jumps, breaking waves, large-scale ships and planing hulls (Mortazavi et al. 2016; Deike et al. 2016; Drazen et al. 2010; Adams et al. 2010; Fu et al. 2010b; Fullerton et al. 2010). Relevant to our effort is the simulation of the transom stern experiments at full and laboratory scale using both iLES and unsteady Reynolds averaged Navier-Stokes (uRANS) flow solvers to compare to measured interface characteristics and statistics (Wyatt et al. 2008; Drazen et al. 2010). In this work, they simulated both a wet and dry transom configuration and quantified the spectrum of the interface fluctuations. However, the work did not provide information on the flow structure or detailed void fraction information.

The only available information that may provide insight regarding the flow field in the mixed region is that of two-dimensional transom experiments (modeled as flow beneath a backward facing step (Maki et al. 2008; Rodriguez-Rodriguez et al. 2011)) and twodimensional supercritical hydraulic jumps (Mortazavi et al. 2016; Chanson 2009; Mossa \& 
Tolve 1998; Hoyt \& Sellin 1989; Lin et al. 2012; Chanson \& Brattberg 2000; Chachereau \& Chanson 2011; Chanson 1995). Collectively, they report that the velocity field contains a mixing shear layer with coherent structures and a peak in the average void fraction in regions of large turbulent shear. Recent non-intrusive measurements of the velocity field in the mixed region (Lin et al. 2012) find that the presence of the large recirculation region in supercritical hydraulic jumps significantly alters the turbulent statistics. DNS simulations (Mortazavi et al. 2016) identify the average interface near the turbulent toe of the jump as a near-stagnant condition below which exists a recirculation region and above which is a jet-like profile. However, the similarity between these two-dimensional flows (both hydraulic jumps and transom sterns) and a three-dimensional wake behind a finite beam transom stern are yet to be understood.

The length scales of interest for this work (laboratory or prototype) are typically many orders of magnitude greater than the Hinze scale $a_{H}$, above which cavity breakup is primarily due to shear and turbulent fluctuations, and below which surface tension and other small-scale effects become important in the bubble dynamics (Hinze 1955). For breaking waves, Deane \& Stokes (2002) report $a_{H} \sim O(1) \mathrm{mm}$, while features in the stern wake could involve length scales $O(10) \mathrm{m}$ or more. Numerically resolving this range would require $>O\left(10^{12}\right)$ grid points, which is computationally prohibitive. Thus, in our iLES, we focus on features greater than $O\left(a_{H}\right)$ and ignore surface tension. The limitation of our results is the grid size $\Delta \gg a_{H}$ and not neglecting sub-Hinze scale surface tension effects. Strictly speaking, we resolve the air entrainment and cavity formation and breakup associated with the large-scale flow, but not the details of the air cavities/bubbles below $O(\Delta)$.

We perform incompressible, two-phase (air-water) iLES on a three-dimensional Cartesian grid using a conservative Volume-of-Fluid (cVOF) method (Weymouth \& Yue 2010). cVOF conserves mass to machine precision, which is a critical component for quantitative prediction of air entrainment. The efficacious boundary data immersion (BDIM) method provides the body representation in the Cartesian grid. Motivated by work such as Drazen et al. (2010), we consider the problem of a canonical transom stern of rectangular cross section (beam $2 B$, draft $D$ ) in deep water idealized as a partially submerged rectangular prism to remove upstream kinematic and geometric influences. To further simplify the problem, we assume uniform and constant-in-time inflow velocity $U$ outside the stern cross-section on the inflow plane. Thus, the parameters that characterize the problem are the half-beam-to-draft ratio $B / D$ and draft Froude number $F r=U / \sqrt{g D}$. We perform iLES for different $B / D$ ratios and dry-transom values of $F r$.

Our simulations obtain the characteristic features of the wake: the converging corner waves originating from the transom; the colliding of these waves aft of the near stern (an event that is geometry dependent) to form the "rooster tail" and the development of a significant three-dimensional, mixed region near the surface; and subsequent formation of the $\mathrm{V}$-shaped diverging-wave wake as the rooster tail widens downstream. We elucidate the mean interface characteristics and define their scaling with ship-scale parameters $B / D$ and $F r$. We explore the mean flow field of the complex turbulent mixed-phase region and identify the presence of a jet and secondary vortex structure below the average interface. We show that the mixed region flow contains a shear region similar to that of two-dimensional hydraulic jumps and two-dimensional stern flows but there is no appreciable evidence that a recirculating region typical of those flows exists immediately aft of the three-dimensional geometry. The breaking waves further downstream in the diverging-wave wake, reintroduce a three-dimensional flow structure that might resemble a roller-bore with strong cross-stream advection on an oblique plane that directly influences the turbulent statistics and air entrainment in this region. 
To identify the entrained air cavities, we develop an algorithm based on the connected component algorithm (CCA) (Samet \& Tamminen 1988) from computer graphics that enables quantification of the air entrainment and cavity size characteristics. We obtain the spatial distribution of the surface entrainment rate, which initially peaks in the converging corner wave region and has a second peak at the end of the rooster tail region with the development of the $\mathrm{V}$-shaped diverging wave region. We observe void fractions of $13 \%$ in the diverging wave region, consistent with laboratory-scale experiments. When we consider the density spectra of cavity sizes $N(r)$ in different regions of the wake, we obtain a power-law spectrum of slope $r^{\beta}$ that is weakly dependent on $B / D$ with $\beta>-4$ when there is active wave breaking.

The outline of this paper is as follows. $\S 2$ details the numerical procedure used to obtain the iLES datasets and outlines the Lagrangian cavity identification algorithm used to determine the entrained air cavity volumes. $\S 3$ defines the canonical ship-wake problem used in this study. $\S 4$ presents the analysis of the wake interface characteristics and the detailed description of the flow structure in the mixed region, including the average velocity and vorticity fields. $\S 5$ details the air entrainment results. $\S 6$ discusses the overall impact of the findings of this paper in the context of large-scale, three-dimensional ship wakes. The appendices contain verification information as well as the parameter study.

\section{Method}

\subsection{Numerical procedure}

We employ implicit large eddy simulation (iLES) of the two (assumed) incompressible fluids, air and water, using a conservative Volume-Of-Fluid method (cVOF) and Boundary Data Immersion method (BDIM) on a Cartesian grid. The three-dimensional velocity field $\vec{u}(\vec{x})$ in a domain $\vec{x}$ obeys the continuity equation $\nabla \cdot \vec{u}=0$ and the momentum equation in the absence of surface tension:

$$
\frac{\partial \vec{u}}{\partial t}+\nabla \cdot(\vec{u} \vec{u})=-\frac{1}{\rho} \nabla p+\frac{\vec{g}}{F r^{2}},
$$

where $p$ is the total pressure field and $\vec{g} / F r^{2}$ the gravitational vector scaled by Froude number Fr. The volume fraction $f$ provides the density $\rho$ using the two fluid densities $\rho_{\text {water }}$ and $\rho_{\text {air }}$ as

$$
\rho(\vec{x})=f(\vec{x}) \rho_{\text {water }}+[1-f(\vec{x})] \rho_{\text {air }} .
$$

We capture the two-fluid interface using a fully conservative three-dimensional volumeof-fluid method, cVOF (Weymouth \& Yue 2010). As in standard VOF methods, $f$ derives from integrating the so-called fluid 'color function', $c(\vec{x})$ defined as 1 or 0 in the water or air, respectively. The volume fraction $f$ within each computational volume $\Omega$ represents the fraction of $c$ within each cell. A simple explicit operator-split update equation ensures conservative transport of the interface via $n$ sweeps over

$$
\Delta f=\frac{\Delta t}{\Delta \Omega} \sum_{d=1}^{n}\left(\Delta_{d} F_{d}+c_{c} \frac{\partial u_{d}}{\partial x_{d}} \Delta \Omega\right)
$$

where $n$ is the number of spatial dimensions, $u_{d}$ the velocity components, $\Delta_{d} F_{d}$ the net fluxes of liquid fluid through the $d$-face of the volume and $c_{c}$ is the cell center value of the color-function given for linear interfaces by

$$
c_{c}=\left\{\begin{array}{cc}
1 & \text { if } \\
0 & \text { else }
\end{array} \quad f>1 / 2 .\right.
$$


As shown in Weymouth \& Yue (2010) the updates of (2.3) along with the Courant restriction on the time step $\Delta t$ of the form

$$
\Delta t \sum_{d=1}^{n}\left|\frac{u_{d}}{\Delta x_{d}}\right|<\frac{1}{2}
$$

guarantees that the transport method is fully conservative for general flows.

We immerse the ship geometry in the Cartesian background grid with BDIM (Weymouth \& Yue 2011), which includes the effect of the solid body on the fluid by convolving the governing equations and interfacial conditions of any solid/fluid system with smooth, finite-width integration kernels. This results in a single governing equation for the complete domain. BDIM's analytic formulation ensures proper treatment of general boundary conditions (Weymouth \& Triantafyllou 2013; Weymouth \& Yue 2011). This work focuses on simulation of stationary bodies immersed in a high Reynolds number flow with free-slip tangential velocity boundary conditions. As such our governing equations are velocity matching for the normal component of velocity $u_{n}$ on the interface

$$
u_{n}\left(\vec{x}_{b}\right)-\mathcal{U}\left(\vec{x}_{b}\right)=0,
$$

where $\mathcal{U}$ is the interface flux, which is zero for solid boundaries. A homogeneous Neumann condition for the tangential velocity components $\left(u_{\sigma}, u_{\tau}\right)$ normal to the interface enforces a free-slip boundary condition on the body

$$
\frac{\partial u_{\sigma}}{\partial n}=0 \quad \text { and } \quad \frac{\partial u_{\tau}}{\partial n}=0 .
$$

Combining (2.1), (2.6) and (2.7) using the BDIM formulation obtains the global meta equation

$$
\vec{u}+\overrightarrow{\mathcal{L}}(\vec{u})=\delta_{e}^{B} \vec{U}+\left[1-\delta_{e}^{B}\right]\left(\vec{u}\left(t_{0}\right)+\int_{t_{0}}^{t_{0}+\Delta t}\left[\vec{r}-\frac{1}{\rho} \nabla p\right] d t\right) .
$$

Here, $\vec{r}$ represents the convection and gravity terms and $\delta_{e}^{B}$ is the zeroth moment of the integration kernel over the body (approximated by a smoothed Heaviside function based on the distance to the body geometry). The left-hand side operator $\mathcal{L}$ is

$$
\overrightarrow{\mathcal{L}}(\vec{u}) \equiv \delta_{e}^{S} \frac{\partial \vec{u}_{\tau}}{\partial n} \hat{\tau}+\delta_{e}^{S} \frac{\partial \vec{u}_{\sigma}}{\partial n} \hat{\sigma}
$$

where $\delta_{e}^{S}$ is the zeroth moment of the integration kernel centered at the nearest point on the interface.

We discretize the governing equations using a staggered-grid finite volume method. The implicit modeling of iLES derives from a flux-limited QUICK treatment of the convective terms. An explicit second-order predictor-corrector method estimates the time integral in (2.8). Hereafter, we normalize all fluid constitutive properties to that of water: $\rho=\rho^{*} / \rho_{\text {water }}$ and $\mu=\mu^{*} / \mu_{\text {water }}$, where ${ }^{*}$ denotes respectively the dimensional density and viscosity of the fluid at a given point. Finally, we determine the pressure via the projection method using the continuity equation. A Multi-Grid solver using ConjugateGradient Smoothing inverts the pressure Poisson matrix. Verification of the method appears in appendix B as well as Weymouth \& Yue (2011), Weymouth \& Yue (2010), Weymouth et al. (2010) and Hendrickson et al. (2012).

\subsection{Air cavity identification}

The air-water interface of high-energy flows possesses non-trivial topological complexity. The forthcoming analysis requires us to identify air entrainment (defined as a cavity 
of air not connected to the bulk air volume), the bulk water volume (defined as the water volume with spray droplets removed and air cavities filled), and the mixed-phase volume (defined as the original $f$ field). To identify spray droplets and air cavities, we develop a method of identifying contiguously connected regions of the three-dimensional, timevarying air volume fraction field $f_{a} \equiv 1-f(\vec{x}, t)$. The method is a modified version of a two-pass standard connected component analysis (CCA) algorithm (Samet \& Tamminen 1988). The algorithm enables the calculation of the individual air cavities $v_{e}^{i}=\int \hat{f}_{a i}(\vec{x}) d \vec{x}$ and effective spherical radius $r_{\mathrm{eff}}^{i}=\left[\frac{3}{4 \pi} v_{e}^{i}\right]^{1 / 3}$. The unique identifiers that result in the CCA algorithm identify the air cavities in $f$ and spray droplets in $f_{a}$ that are either filled or removed to calculate the water bulk region in $\S 4.2$. Appendix A further discusses the details of this Lagrangian analysis technique.

\section{Simulation of a canonical 3D dry transom stern}

The canonical problem we consider is the flow behind a rectangular transom stern of half-beam $B$ and still water draft $D$. We scale by the draft $D$ and the uniform steady inflow velocity $U$. The draft Froude number $\mathrm{Fr}=U / \sqrt{g D}$ characterizes this problem. The cases considered are at sufficiently high $F r$ to correspond to dry transom conditions. Recent experiments of Drazen et al. (2010) using a Model 5674 geometry with draft of $D=0.305 \mathrm{~m}$ showed that transition to dry transom conditions occurred between 7 $8 \mathrm{kts}$ or $\mathrm{Fr}=2.38$ at laboratory scale. Performing many iLES over a range of $F r$ $2.38 \leqslant F r \leqslant 3$ and half-beam-to-draft $B / D$ ratios $1 \leqslant B / D \leqslant 2.1$, we find that the results are qualitatively similar, and can be described with a set of representative cases $\mathrm{A}, \mathrm{B}$, and $\mathrm{C}$ (details in table 1).

The boundary conditions for the Cartesian grid iLES (figure 1) are as follows: (i) uniform inflow velocity $U$ on the inlet plane except its intersection with the rectangular body geometry where the body boundary conditions are enforced as described in $\S 2.1$; (ii) zero-gradient extrapolations on the lateral boundaries; (iii) symmetry planes for the top and bottom boundaries; and (iv) mass conserving exit condition far downstream. We subdivide the computational domain into a high-resolution inner interrogation domain of constant grid volumes $\delta \Omega=\Delta^{3}$ that encompasses the near region behind the stern. Our interest is the large-scale features of the air-entraining turbulent wake extending horizontally $O(10 D)$ behind the stern. Thus, we size the interrogation domain to be $L_{x} \times L_{y} \times L_{z}=13 D \times 10 D \times 3 D$ where $x, y, z$ are respectively the longitudinal, transverse and vertical dimensions. The interrogation grid resolution is $\Delta=D / 64$. This is sufficient to resolve features an order smaller than $D$, but the numerical Hinze scale $a_{N}=\Delta \sim 1 \mathrm{~cm}$ is still substantially greater than the physical Hinze scale $a_{H}$ for realistic laboratory-scale (and certainly full-scale) stern experiments (Drazen et al. 2010; Terrill \& Fu 2008; Fu et al. 2006). A buffer domain that employs algebraic grid stretching surrounds the interrogation domain, extending the outer boundaries and increasing the numerical damping in the transverse and far-stream regions. The entire Cartesian grid (interrogation and buffer domain) is $N_{x} \times N_{y} \times N_{z}=1024 \times 896 \times 256=2.35 \times 10^{8}$ spatial grid cells.

We initiate the simulation by linearly increasing the inflow velocity over a period of $t=T U / D=10$. Once at a quasi-steady state, we sample the interrogation domain to obtain time-averaged statistics over $80 \leqslant t \leqslant 120$ with a sampling rate at $d t=0.01$. All averages reported herein are temporal averages over this period of time with this sample rate. Table 1 reports the median $\nu_{e}^{m}$ and third-quartile $\nu_{e}^{3 r d}$ value of the iLES effective viscosity (defined in appendix B).

We utilize three different analysis techniques throughout part 1 and part 2 . In $\S 4.2$, we 
Case $\operatorname{Fr} \quad B / D\left(\nu_{e}^{m}\right)^{-1}\left(\nu_{e}^{3 r d}\right)^{-1}$

$\begin{array}{lllll}\mathrm{A} & 2.53 & 1.0 & 3760 & 5290 \\ \mathrm{~B} & 2.53 & 1.25 & 3681 & 5290 \\ \mathrm{C} & 2.53 & 1.77 & 4142 & 5064\end{array}$

TABLE 1. Parameters for representative cases A, B, and C measured within the interrogation domain. $\nu_{e}^{m}$ and $\nu_{e}^{3 r d}$ are the median and third-quartile values of the iLES effective viscosity (defined in appendix B). The time step $\Delta t=1.875 \times 10^{-3}$.

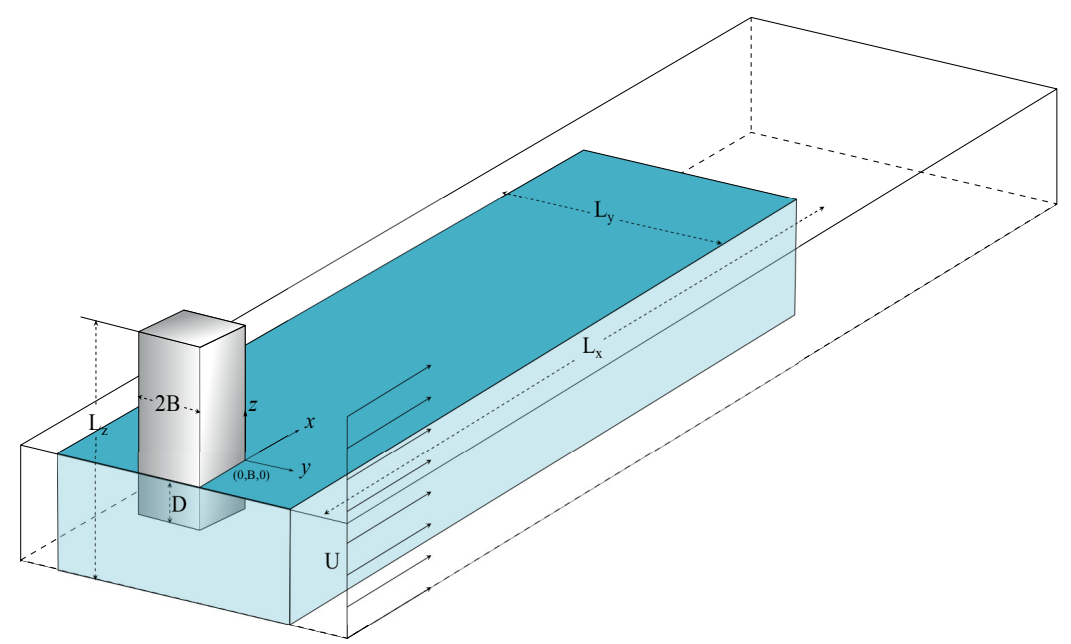

FiguRE 1. Schematic of the canonical stern geometry. Blue area: the high-resolution, interrogation domain $L_{x} \times L_{y} \times L_{z}=13 D \times 10 D \times 3 D$.

use the modified CCA of $\S 2.2$ and appendix A to isolate the connected isosurface that represents the unbroken air-water interface and water bulk region by removing both air bubbles and spray droplets. We use a temporal average of the resulting bulk fraction $\overline{f_{b}}$ to identify the surface region $0<\overline{f_{b}}<1$ incorporates only surface fluctuations and $\overline{f_{b}}=0.5$ represents the mean interface. In $\S 4.3$, we use a purely Eulerian framework with unconditioned time averages where $0<\bar{\rho}<1$ represents the mixed-phase region (or mixed region) and incorporates surface fluctuations, entrainment and spray. Part 2 uses this framework exclusively and uses the turbulent mass flux $\overline{\rho u_{i}^{\prime}}$ to represent all of these physical processes as a single vector quantity. In $\S 5$, we adopt a Lagrangian framework to focus solely on the air entrainment. Using the modified CCA to identify air bubbles, we calculate the bubble statistics and define the entrainment envelope from a temporal average of $v_{e}$ on the Cartesian grid. Figure 2 shows the conceptual difference between the entrainment envelope, mixed region, and surface region using a transverse cut in the far wake (looking upstream). Note that the lines for $\bar{f}=\overline{f_{b}}=0.5$ coincide, as expected.

\section{Wake characteristics and flow features}

\subsection{General wake features}

Figures 3, 4 and 5 shows the isosurface of the instantaneous volume fraction $f=0.5$ for respectively case A, B and C. All cases have three distinct characteristic regions in the surface of the wake, which we refer to hereafter as the converging corner waves $(\mathrm{CCW})$, 


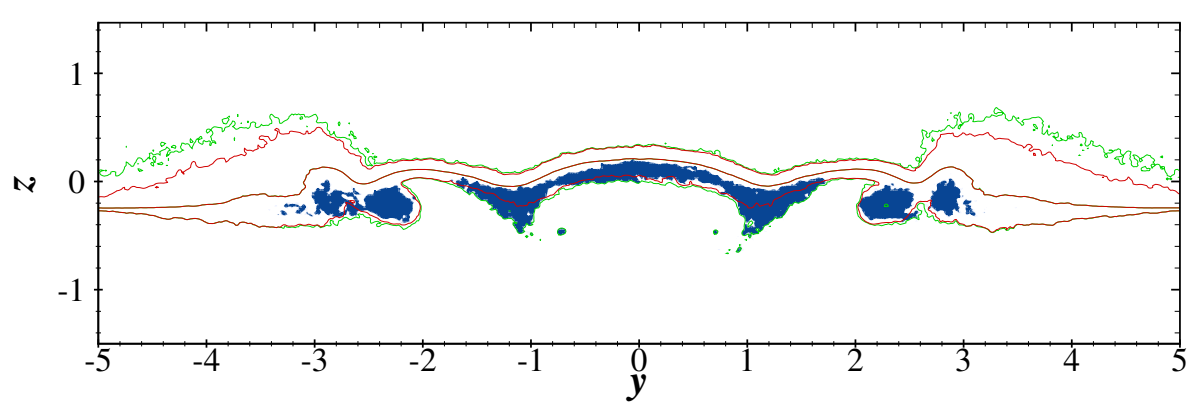

FIGURE 2. Comparison of entrainment envelope, surface region and the mixed region for a transverse cuts at $x=11.5$. Green lines $\bar{f}$ and red lines $\overline{f_{b}}=(0.01,0.5,0.99)$ from top to bottom within each figure, respectively. Blue region is $\overline{v_{e}} \geqslant 0.01$. Data is case B.

the rooster tail (RT), and the diverging wave (DW) regions. The first region contains a large depression behind the dry stern with ridges that rise from the lower corner. These ridges, or CCW, angle in towards the centerline of the ship. After the $\mathrm{CCW}$ region is the RT, the length of which decreases with $B / D$. The surface of the RT is rough with many small ligaments and spray. The RT region widens to form the beginning of the DW train behind the stern. Here, the center of the wake surface becomes smoother and the edges contain very fine structures and quasi-steady breakers. Scars on the interface (indicated by the dark features angling away from the centerline) indicate regions of wave overturning and air entrainment similar to that observed by (for example) Dong et al. (1997) and Olivieri et al. (2007) for overturning bow waves.

While each wake contains these distinct characteristic regions, there are overall differences in the wake between cases A/B and case C. For case A and B, the CCW collide on the ship centerline before overturning and entraining a fully-formed air cavity. The physics at this collision point represent two impacting jets and serve as a source of significant spray. For case C, the $\mathrm{CCW}$ have ample time to break and entrain air before the formation of the RT. Because of this, the RT in case $\mathrm{C}$ is smoother with less spray and ligaments than cases $\mathrm{A}$ and $\mathrm{B}$.

\subsection{Wake characteristics and scaling}

We obtain a cleaner depiction of the instantaneous surface by utilizing the cavity identification algorithm of $\S 2.2$ to identify the air cavities and spray droplets and either filling them (if air) or removing them (if water). The resulting instantaneous bulk volume fraction $f_{b}$ is the result. Figure 6 shows the time-averaged isosurface of $f_{b}$ corresponding to $\overline{f_{b}}=0.5$ for case $\mathrm{A}$ (case $\mathrm{B}$ is similar). The location where the CCW collide on the centerline $x_{c}$ appears different when viewed from above (figure 6a) and below (figure 6b) due to the multivalued nature of the interface. The RT is present for $x>x_{c}$. At a point further downstream, the waves radiate away from the centerline and form a divergent wave pattern.

The experiments of Martínez-Legazpi et al. (2013) established that the trajectory of the wavefront emanating from the corner of a partially submerged vertical plate is ballistic by considering the impact point of the corner wave on the surface downstream of the plate. A similar potential energy argument for the stern geometry obtains the scaling of $x_{c}$. Consider the flow leaving the bottom corner of the stern at $x=0$ with speed $\left(U, v_{0}, w_{0}\right)$. Assuming the velocity in the $y-z$ plane is gravity driven, the $y-z$ plane 
(a)

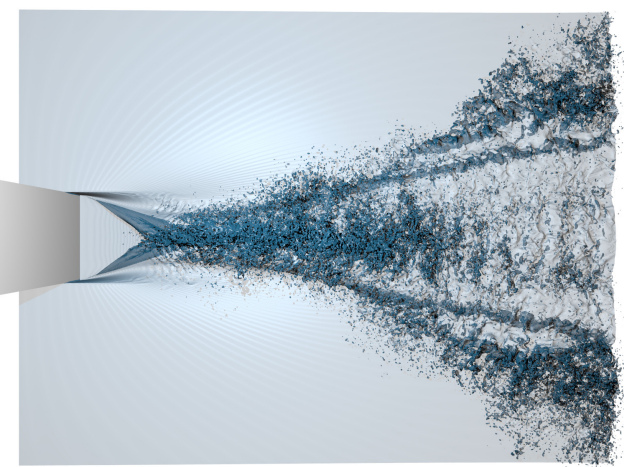

(b)

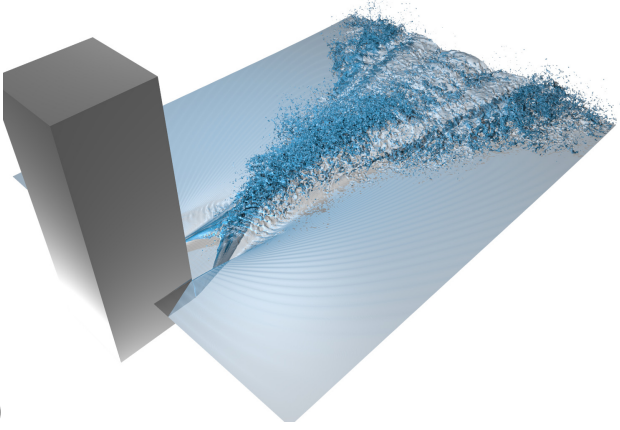

FiguRE 3. Instantaneous isosurface of volume fraction $f=0.5$ for case A within the interrogation domain. Flow is from left to right $(+x)$. (a) viewed from above; (b) viewed from the side. Surface is rendered partially transparent. Dark regions indicate multivalued surface, spray or entrainment. Corresponding movie included as supplementary material.

(a)

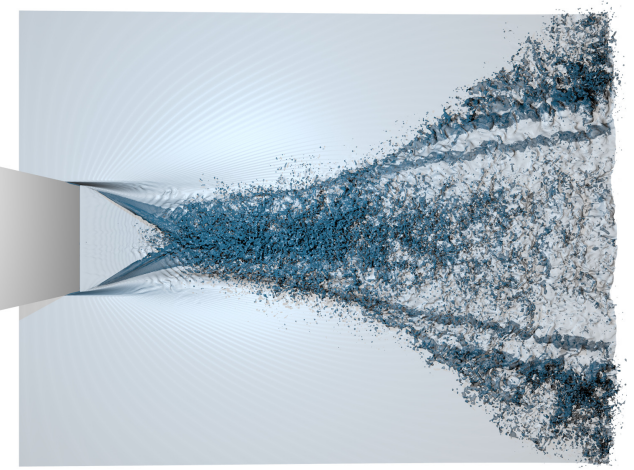

(b)

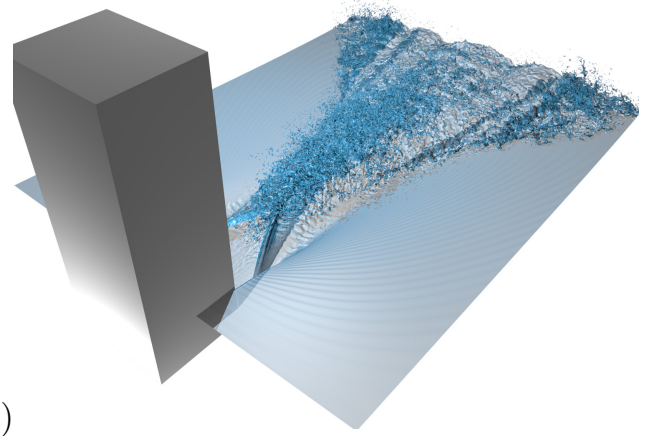

Figure 4. As in figure 3 for case B. Corresponding movie included as supplementary material.

(a)

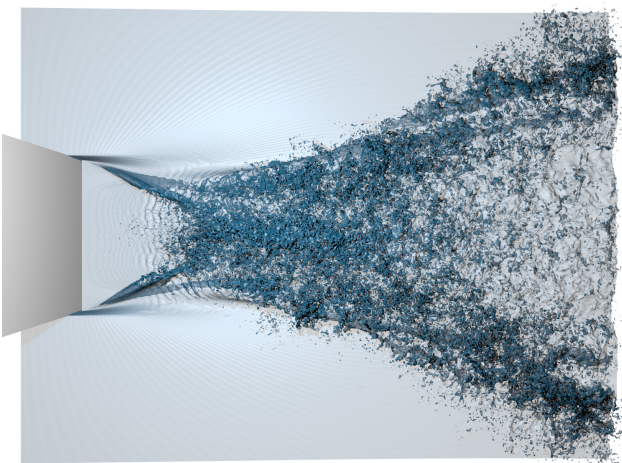

FiguRE 5. As in figure 3 for case C. Corresponding movie included as supplementary material. (b)

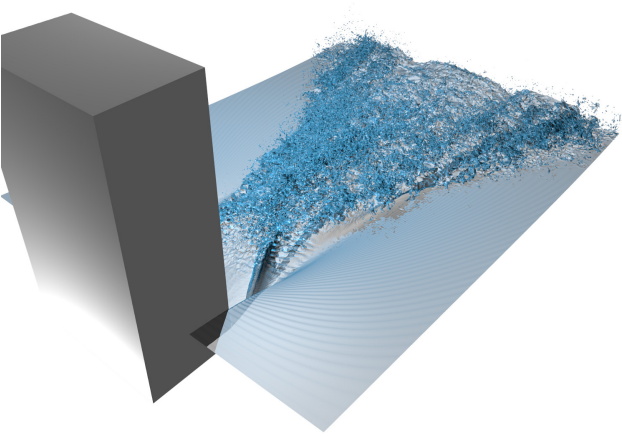




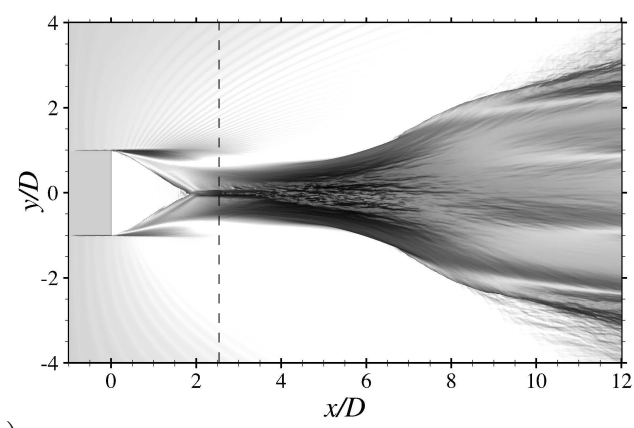

(a)

Figure 6. Average interface $\overline{f_{b}}=0.5$ case A. (a) viewed from above and (b) below. Contours represent elevation $z$ (light to dark, negative to positive). - - $x_{c}$. Note that the "ripples" on the interface near the inlet are an artifact of the visualization of the isosurface and not present in the simulation.

velocity $\left(v_{0}, w_{0}\right)=\sqrt{2 g D}\left(\cos \phi_{c}, \sin \phi_{c}\right)$ where $\phi_{c}$ is the angle of the projectile motion in the $y-z$ plane (see figure $7 \mathrm{a}$ ). The time for a particle to travel the distance $B$ to the centerline, assuming $v_{0}$ and $U$ constant, provides the location where the corner waves meet and the angle of the waves in the $x-y$ plane $\theta_{c}$ as:

$$
x_{c}=F r B \alpha^{-1} \text { and } \tan \theta_{c}=\alpha F r^{-1},
$$

where $\alpha=\sqrt{2} \cos \phi_{c}$. Thus for a dry rectangular transom stern, $F r, B$ and $\phi_{c}$ completely determine the location of the collision of the CCW and the beginning of the RT. This ballistic behavior and gravity-driven scaling of $x_{c}$ is also consistent with supercritical channel expansion flows (Rouse et al. 1949, when $\phi_{c}=0$ ).

To measure the location of $x_{c}$, we first determine the location of the interface without interpolation by defining the integrated height function $H(x, y) \equiv \int_{z} \overline{f_{b}} d z$. We then determine the transverse location $Y_{m}(x)$ of the local maximum at each $x$ of $H(y ; x)$. A projection of a linear fit of $Y_{m}(x)$ provides $x_{c}$ such that $Y_{m}\left(x_{c}\right)=0$ and the slope of the linear fit provides $\theta_{c}$ (see figure $7 \mathrm{~b}$ ). This technique works best when $\overline{f_{b}}$ is not multi-valued, thus the linear fit of $Y_{m}(x)$ is taken from the region immediately aft of the stern. To obtain $\phi_{c}$, we use a linear fit of the same local maximums in the $y-z$ plane. The measured initial value of $\phi_{c}$ is consistent with potential flow theory in the absence of gravity, where $\phi_{c}=\pi / 4$ at very early times (Martínez-Legazpi et al. 2015). The first three columns of table 2 are near unity in accordance with the ballistic result of (4.1). For cases at a constant Froude number (as these are), if the potential energy of the free-surface jump proportional to $D$ is the only driving mechanism, then CCW features should be the same and the changes in $B / D$ only moderate the value of $x_{c}$. The data in table 2 establishes this and a small parametric study confirms this scaling and driving mechanism for a broader range of $B / D$ and $F r$ (see appendix $\mathrm{C}$ ).

To understand the scaling of the far wake geometric parameters, we define the wake angle $\theta_{w}$ (see figure $7 \mathrm{~b}$ ) as the angle of the beginning of the DW region. We measure this angle by performing a linear fit of the transverse locations $Y_{0}(x)$ of the zero-crossing of $H(y ; x)-H_{s w l}$, where $H_{s w l}$ is the static water line. For convenience, we measure $\theta_{w}$ at the streamwise point $x_{B}$ where $Y_{0}$ crosses the $y=B / D$ plane. As seen in table 2 , the wake angle shows no significant geometry dependence.

Figure 8 shows the centerline interface height $\Delta h=H(0 ; x)-H_{\text {swl }}$ (relative to the bottom of the stern) for all three cases, plotted using the ballistic scaling $\tilde{x}=$ 
Flow and Entrainment in 3D Dry Transom Wakes

$\begin{array}{llllccc}\text { Case } & \tan \phi_{c} & \frac{x_{c} \alpha}{F r B} & \frac{\tan \theta_{c} F r}{\alpha} & \frac{\tan \theta_{w}}{F r} & \Delta h_{\max } & \frac{x_{B}}{F r} \\ \mathrm{~A} & 0.99 & 1.07 & 1.01 & 0.53 & 1.24 & 2.68 \\ \mathrm{~B} & 0.99 & 1.10 & 1.01 & 0.47 & 1.20 & 2.87 \\ \mathrm{C} & 1.17^{*} & 0.92^{*} & 1.03^{*} & 0.52 & 1.15 & 3.06\end{array}$

TABLE 2. Average wake geometry characteristics. $\alpha \equiv \sqrt{2} \cos \phi_{c}$. ${ }^{*}$ Data measured by linear extrapolation of $Y_{m}(x)$ for $x<1$ due to the multivalued nature of the interface in the CCW region.

(a)

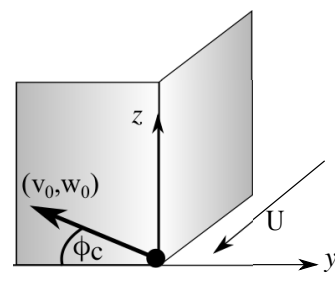

(b)

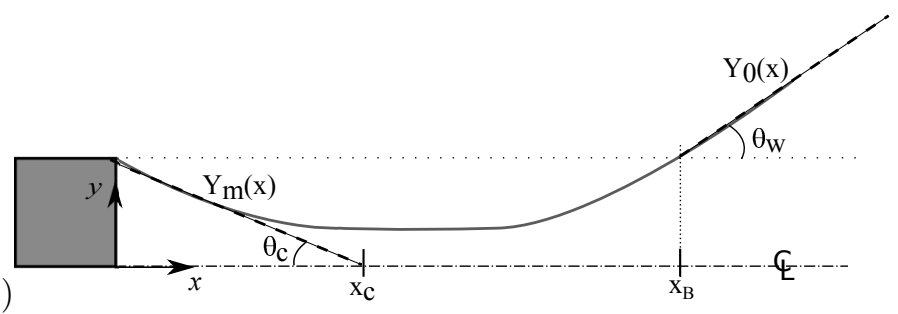

FiguRE 7. (a) Schematic of the ballistic projectile motion viewed looking upstream and definition of $\phi_{c}$. (b) Schematic of wake topology viewed from above showing the definitions of $Y_{m}, Y_{0}, \theta_{c}$ and $\theta_{w}$ (displaying only $y>0$ for clarity).

$x \alpha(B F r)^{-1}$. The centerline interface height assumes the same slope for each case until $\tilde{x} \approx 1=\tilde{x}_{c}$. For $\tilde{x}>\tilde{x}_{c}$, the profiles continue to a maximum value of $\Delta h$ (see table 2 ) and then decreases. For cases $A$ and $C$, the maximum location occurs within our definition of RT, where we measure $\theta_{w}$. Case B has an initial local maximum at $\tilde{x}_{c}$ and then a secondary maximum. Inspection of the average interface $\overline{f_{b}}=0.5$ shows that this location contains both the collision and breaking of the CCW. The $\Delta h_{\max }$ for each case is $\approx 1.2$, which is similar to the transom stern experiments of Drazen et al. (2010) (see filled symbols in figure 8).

It is tempting to compare the average interface structure on the ship centerline of a dry transom stern to low Froude number two-dimensional hydraulic jumps (e.g. Mortazavi et al. 2016; Lin et al. 2012)) and two-dimensional sterns (Maki et al. 2008; RodriguezRodriguez et al. 2011). However, the average interface of a two-dimensional hydraulic jump increases sharply over a distance twice the fluid initial depth $h_{1}$ and then asymptotically increases to an analytic value of $\left(-1+\sqrt{1+8 F r_{h_{1}}^{2}}\right) / 2$ (Mortazavi et al. 2016). The overall behavior of the centerline interface height of the three-dimensional stern (i.e. a steep increase, a local maximum and then a decrease) is different. Additionally, the $\Delta h_{\max }$ value of 1.2 is significantly less than the analytic value for these Froude numbers $\left(\Delta h_{\max } \sim 2.1\right.$ if $\left.D \sim h_{1}\right)$.

\subsection{Average flow structure}

Figure 9 shows a visualization of the three-dimensional average flow structure for case $\mathrm{A}$ where the CCW collide on the centerline (figure 9a-b) and the case $\mathrm{C}$ where the CCW fully overturn before the formation of the RT (figure 9c-d). The CCW region for the narrower geometry focuses the flow to the collision point $x_{c}$, which is still present (but weaker) for case $\mathrm{C}$ due to the fully overturning CCW. The color of the average streamlines represents $\bar{\rho}$ at that point which varies along the streamline. From the mean mass conservation equation for a variable density flow, $\partial \bar{\rho} / \partial t+\partial\left(\bar{\rho} \bar{U}_{k}\right) / \partial x_{k}=-\partial\left(\overline{\rho u_{k}^{\prime}}\right) / \partial x_{k}$, we see that there is a source term on the right-hand side equal to the divergence of the turbulent 


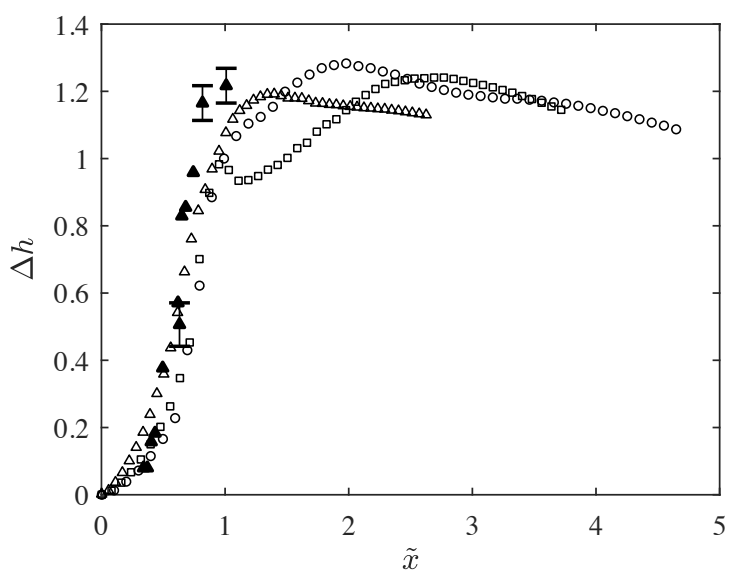

Figure 8. Centerline interface height $H(y=0 ; \tilde{x})$ (relative to the bottom of the stern located at $z=-1$. $\circ$ case A; $\square$ case B; $\triangle$ case C; and $\boldsymbol{\Delta}$ Drazen et al. (2010) 8 knot case. Every 16th point of simulation data plotted for clarity.

(a)

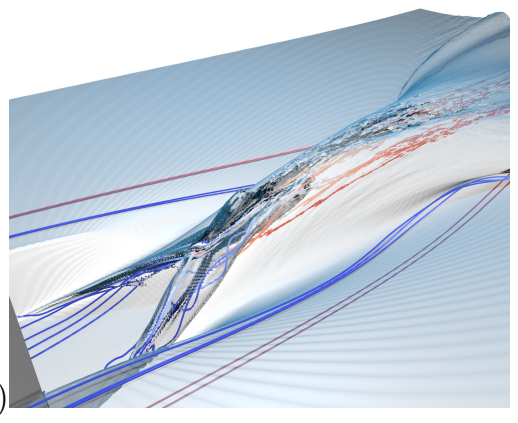

(c)

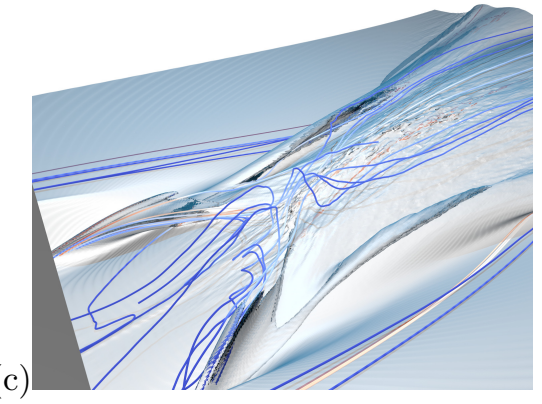

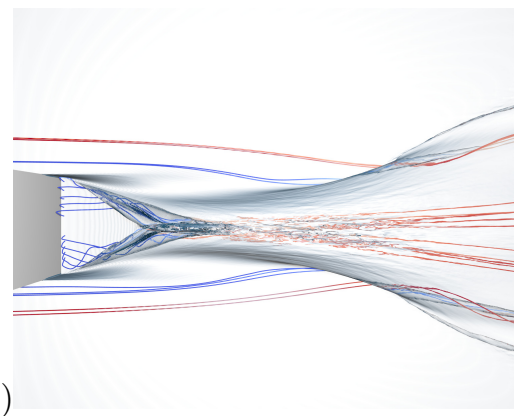

(b)

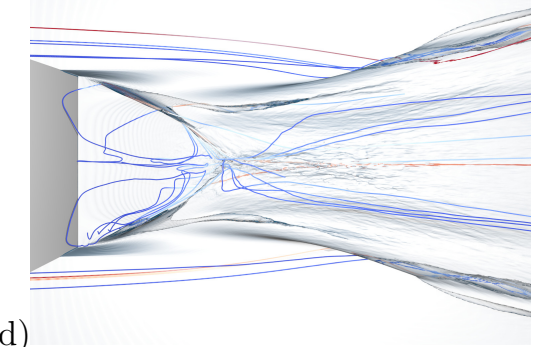

Figure 9. Visualization of average stream lines with average interface for (a) case A side view, (b) case A top view, (c) case C side view, (d) case C top view. Lines colored by $\bar{\rho}$. Red: $\bar{\rho}=1.0$ and blue: $\bar{\rho}=0.001$

mass flux $\overline{\rho u_{k}^{\prime}}$ (see part 2 Hendrickson \& Yue submitted). The change in $\bar{\rho}$ along an average streamline thus reflects the source term associated with the turbulent mass flux $\overline{\rho u_{k}^{\prime}}$.

Figure 10 shows transverse cuts of the average planar flow field $(\bar{v}, \bar{w})$ and magnitude of the average velocity for cases $\mathrm{A}$ and $\mathrm{C}$ within the three different wake regions. Figures 10a-b represent the CCW region where the waves collide at the centerline or overturn. For the narrow geometry of case A near $\tilde{x}=1$ (see figure 10a), the planar flow field shows 
two jets colliding and shooting liquid in the vertical direction. The spray field (mainly the region above the mean interface) is significant. Figure 10b shows the overturning $\mathrm{CCW}$ splash up of the jet for the wider geometry of case $\mathrm{C}$ at $\tilde{x}=0.64$. Figures $10 \mathrm{c}-\mathrm{d}$ represent locations within the RT. Here, both wakes contain a velocity deficit below the mean interface at the centerline $y \sim 0$. This deficit is due to the presence of the upstream corner wave collision. The average planar velocity vectors show the turning of the velocity field away from the center plane as part of the formation of the downstream DW region. The spray field contributes the most to the extent of the mixed region at this streamwise location in the RT. The depth of the mixed region below the mean interface increases in the RT due to the presence of air entrainment. Figures 10e-f represent the DW region $10 D$ from the stern for both geometries. For case A specifically, we observe the primary $\overline{\omega_{x}}$ vortical structures (see inset) caused by the upstream breaking. For both cases, the mixed region widens as it encompasses surface fluctuations, spray and air entrainment. Of note is the confinement of the mixed region to a small vertical extent below the mean interface at this wake distance.

Figure 11 shows select profiles of the averaged planar velocity field $(\bar{u}, \bar{w})$ and corresponding mean transverse vorticity $\overline{\omega_{y}}$ profiles on the center plane. In the CCW region (at $\tilde{x}_{c}$ ), a shear layer appears in $\bar{u}$ at the transition from the bulk water region to the mixed-phase region. At this $\mathrm{x}$ location, $\bar{u}$ also exhibits a jet flow above the shear layer. The jet is strongest for cases where the CCW collide on the center plane (e.g. figure 11a) but still present for the widest geometry (figure 11b). There is also a positive $\bar{w}$ at this location near the mean interface $\bar{\rho}=0.5$, or $z-z_{i}=0$. The mean transverse vorticity (see corresponding figures 11e-f) shows a strong negative peak at the location of the shear layer. A secondary vortex structure exists above this primary structure (below the average interface) as a consequence of the aforementioned jet.

In the DW region on the ship center plane (see figures $11 \mathrm{c}-\mathrm{d}$ and $11 \mathrm{~g}-\mathrm{h}$ ), the jet is no longer present and the velocity profile on the ship center plane in the mixed region is a wake deficit with minimal mean vertical velocity. The far wake location only has the primary vorticity associated with the wake deficit profile. Inspection of the flow field at $y$ locations off of the centerline that intersects breaking waves (in the CCW for case $\mathrm{C}$ and the DW for all cases) contain a strong jet flow with secondary vortex structure similar to that shown in figure 11a-b (not shown).

We comment on the difference between this mixed-region flow structure to those of twodimensional hydraulic jumps. In some sense, the only qualitative similarity is the presence of the shear layer at the transition between the bulk water and mixed-phase regions. The most significant difference between these flows is there is no appreciable evidence for the presence of a primary vortex structure situated above a dividing streamline immediately downstream of the (three-dimensional) stern geometry. In the three-dimensional stern flow, the presence of the CCW introduces a strong three-dimensional flow structure in the mixed region. The resulting jet and corresponding secondary vortex of this threedimensional flow are qualitatively different from the two-dimensional model of a roller above a dividing streamline. Further downstream of the stern, the center plane flow is a wake deficit profile. The presence of three-dimensional breaking in the DW region reintroduces the strong three-dimensional structure to the flow similar to those observed in the CCW. In the DW region, the three-dimensional flow might resemble roller-bore structure in an oblique plane but with the presence of a strong cross-stream advection. Its presence will significantly alter the turbulent statistics in this region (evidence of this in part $2, \S 3)$. As shown in the forthcoming section, the three-dimensional breaking in DW is also associated with enhanced air entrainment in the far wake. 
(a)

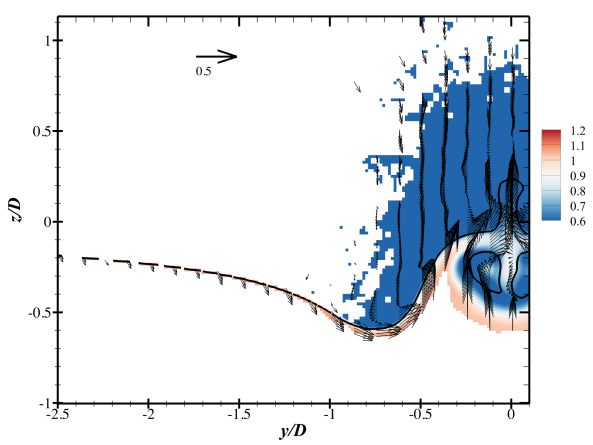

(b)

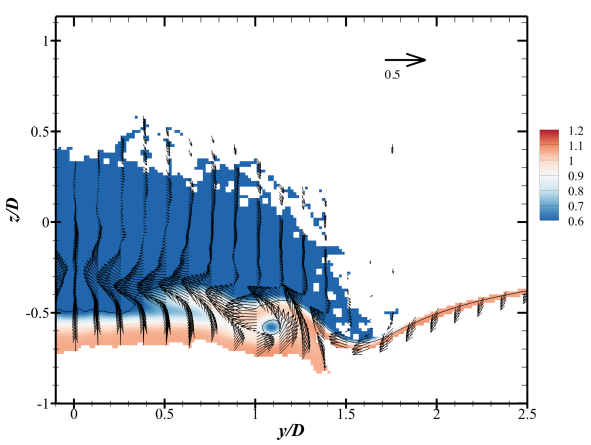

(d)
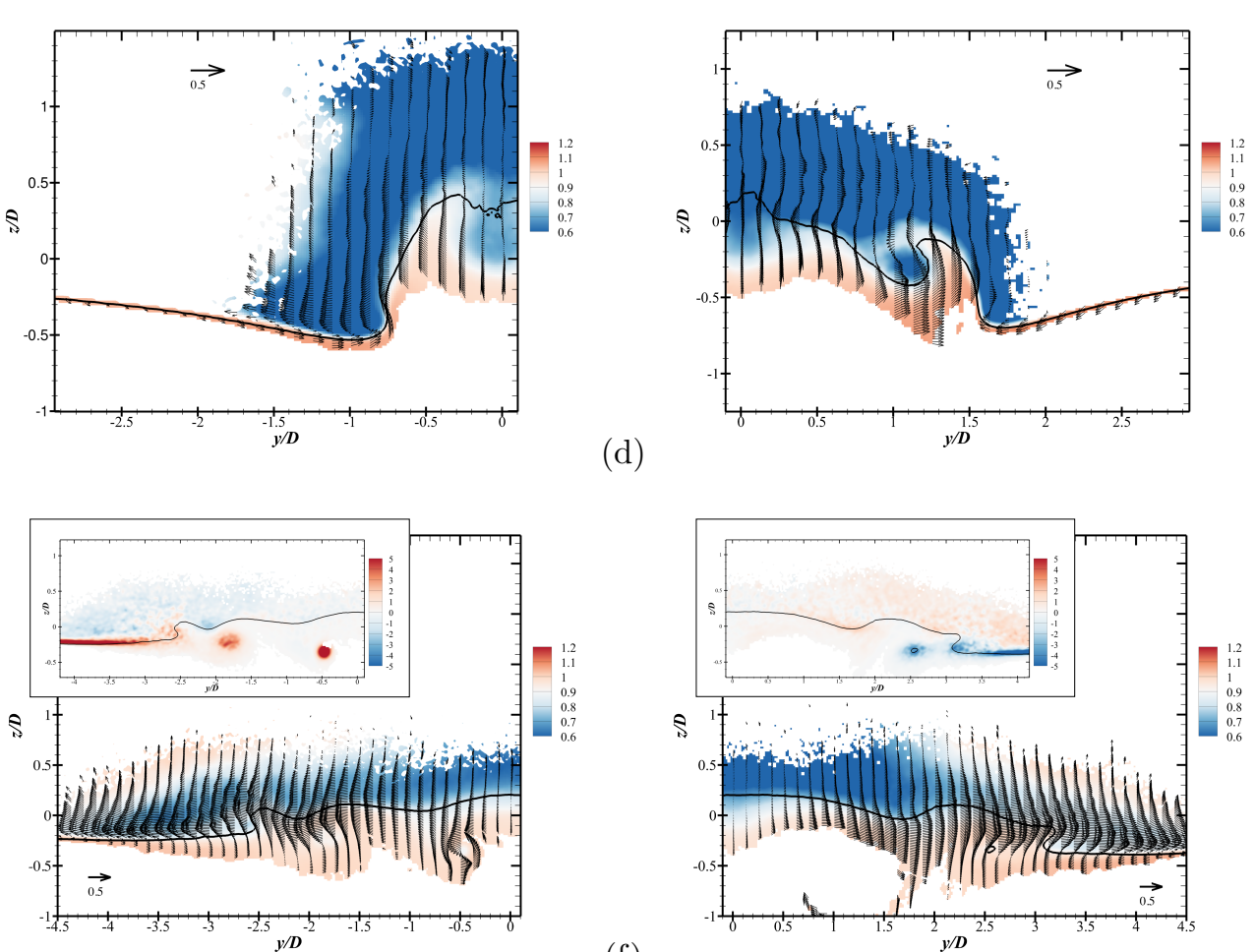

(c)

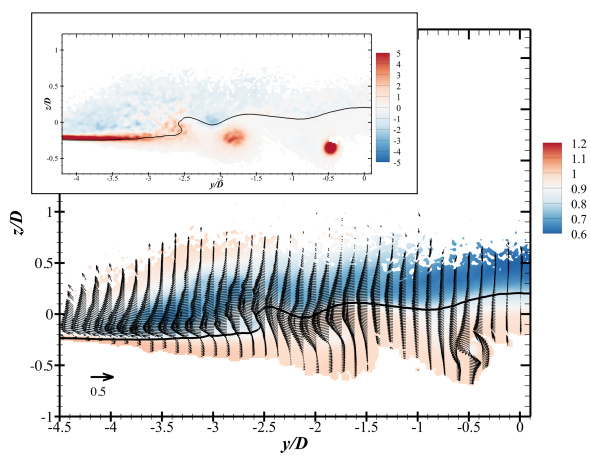

(e)

Figure 10. Transverse cuts of average planar velocity $(\bar{v}, \bar{w})$. Contours represent magnitude of average velocity $\bar{u}$. Black line represents $\bar{\rho}=0.5$. CCW: (a) Case A $\tilde{x}=1$ and (b) case C $\tilde{x}=0.64$; $\mathrm{RT}$ : (c) case A $\tilde{x}=1.96$; (d) case C $\tilde{x}=1.12$; DW: (e) case A $\tilde{x}=3.95$; and (f) case C $\tilde{x}=2.23$. Inset in (e) and (f) is corresponding average streamwise vorticity $\overline{\omega_{x}}$. Vectors shown (every 8 points) within the mixed region $0.01<\bar{\rho}<0.99$.

\section{Air entrainment}

This section adopts a Lagrangian description of the density (or volume fraction) field using the cavity identification algorithm described in $\S 2.2$ to determine the characteristics of the large-scale air entrainment to establish a foundation for bubble source modeling in the near wake of a stern. The Eulerian definition of the mixed region of $\S 4.3$ is such that a variation in density at a single point encompasses all of the physical effects such as surface 

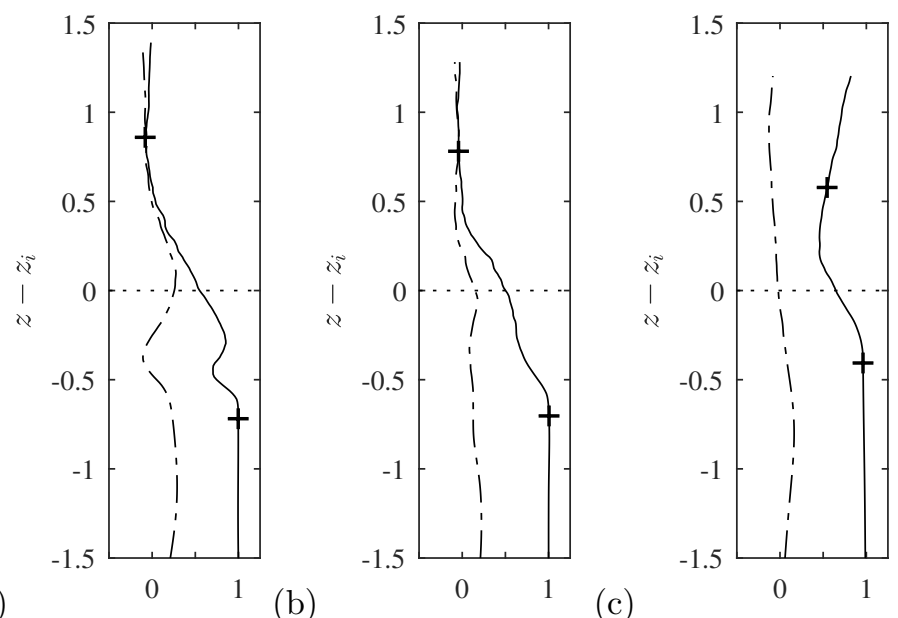

(a)

(b)
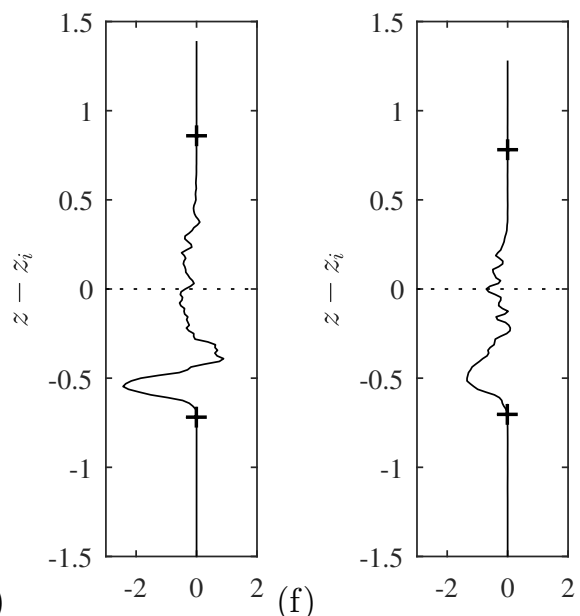

(c)

(d)
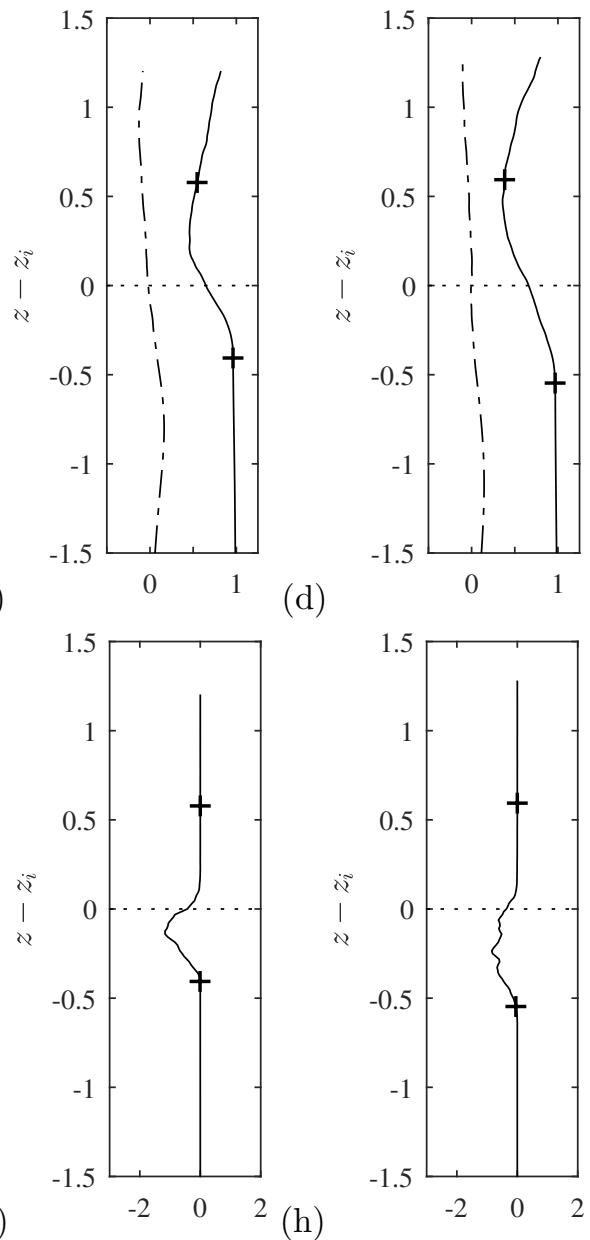

FIgURE 11. Ship centerline wake profiles at select locations as a function of $z-z_{i}$, where $z_{i}$ is the location of $\bar{\rho}=0.5$. (a-d) $-\bar{u}(z) ;--\bar{w}(z)$; and $+\bar{\rho}=(0.01,0.99)$. (e-h) $-\bar{\rho} \overline{\omega_{y}}$ and $+\bar{\rho}=(0.01,0.99)$. (a,e) case B $\tilde{x}=1 ;(\mathrm{b}, \mathrm{f})$ case $\mathrm{C} \tilde{x}=1 ;(\mathrm{c}, \mathrm{g})$ case B $\tilde{x}=3 ;(\mathrm{d}, \mathrm{h})$ case $\mathrm{C} \tilde{x}=2.1$.

fluctuations, spray and air entrainment. Understanding the mean flow and turbulent characteristics in the mixed region using the Eulerian framework enables the development of incompressible highly variable turbulence models and is left for part 2 (Hendrickson \& Yue submitted). Using the following Lagrangian techniques, analysis of the spatial and size distribution of the actual air cavities provides a physical understanding of only the entrainment.

\subsection{Volumetric entrainment and surface entrainment rate}

Let $v_{e}(\vec{x}, t)$ represent the fractional volume of the entrained air at any given point based on the individual air cavities calculated from the cavity identification algorithm of $\S 2.2$. Figure 12 shows a visualization of the average entrainment $\overline{v_{e}}(\vec{x})$ for cases $\mathrm{A}$ (case B is similar) and $\mathrm{C}$. The entrainment is concentrated in regions of breaking waves and in the rooster tail region. For cases $\mathrm{A}$ and $\mathrm{B}$, the $x_{c}$ location is not a strong a source of entrained. For example, the maximum $\overline{v_{e}}$ in this region is 0.04 for case A. We reiterate here that the jet impact on the ship centerline generates a large positive vertical 


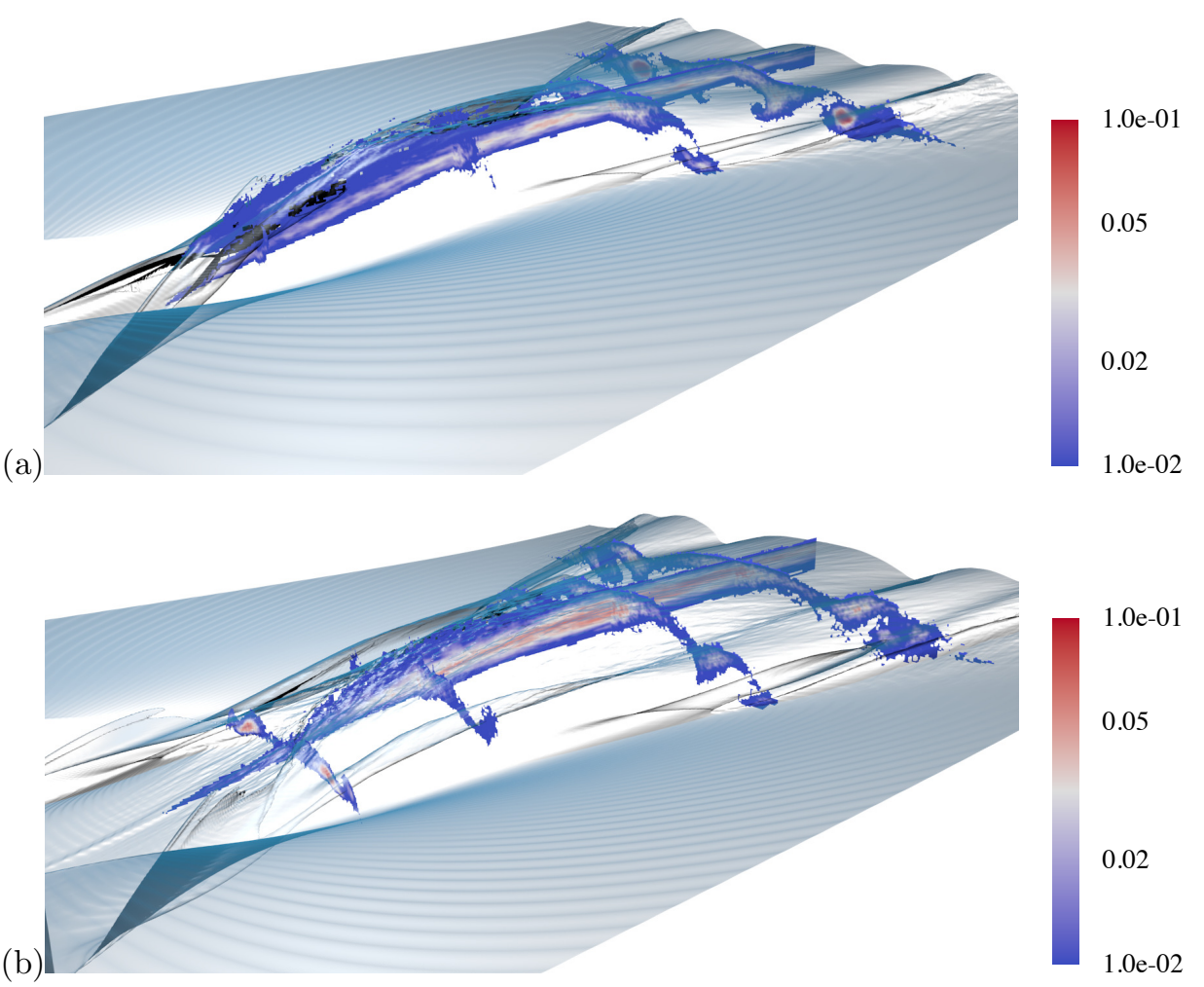

FIgURE 12. Visualization of average entrainment $\overline{v_{e}}(\vec{x})$ at streamwise locations for (a) case A and (b) case C. Transparent isosurface is the average interface $\bar{f}_{b}=0.5$.

$\begin{array}{lccccc}\text { Case } & \int \overline{v_{e}} d \forall / B & \hat{x}^{*} & \overline{\mathcal{S}}\left(\hat{x}^{*}\right) / B & \hat{x}^{d} & \overline{\mathcal{S}}\left(\hat{x}^{d}\right) / B \\ \mathrm{~A} & 0.14 & 1.00 & 1.64 \mathrm{E}-3 & 3.05 & 8.01 \mathrm{E}-3 \\ \mathrm{~B} & 0.09 & 0.94 & 1.40 \mathrm{E}-3 & 2.88 & 5.03 \mathrm{E}-3 \\ \mathrm{C} & 0.09 & 0.91 & 3.31 \mathrm{E}-3 & 2.58 & 2.81 \mathrm{E}-3\end{array}$

TABLE 3. Average entrainment characteristics. $\hat{x}=x / F r$.

velocity and significant spray (see figure 10a-b). The entrainment by the entrapment of an air pocket due to the overturning waves in the $\mathrm{CCW}$ in case $\mathrm{C}$ is more than twice as large with maximum $\overline{v_{e}}=0.09$. This is also true in the $\mathrm{DW}$ region where the maximum values $\overline{v_{e}}$ are the largest within the wake: 0.20 for case $\mathrm{A}$ and 0.13 for cases $\mathrm{B}$ and $\mathrm{C}$. The maximum penetration depth of the entrainment envelope (defined as $\overline{v_{e}} \geqslant 0.01$ ) is $-0.85<z<-0.69$ relative to the still water line for both the DW and RT regions in all three cases.

We define the entrained air volume per unit distance as $\overline{V_{e}}(x)=\int_{-\infty}^{\infty} d y \int_{-\infty}^{\infty} d z \overline{v_{e}}(\vec{x})$. Figure 13a shows $\overline{V_{e}}(\hat{x})$ (normalized by the half-beam $B$ for a simple length scale) for all three cases, where $\hat{x}=x / F r$ scales the streamwise distance with the ship speed. The initial peak of entrained volume occurs near $\hat{x} \gtrsim 1$ for all three cases. The entrainment increases in the DW $\hat{x} \gtrsim 2.5$. Table 3 shows the total amount of entrained volume per unit half-beam. The narrowest geometry (case A) entrains the most air while case B and 

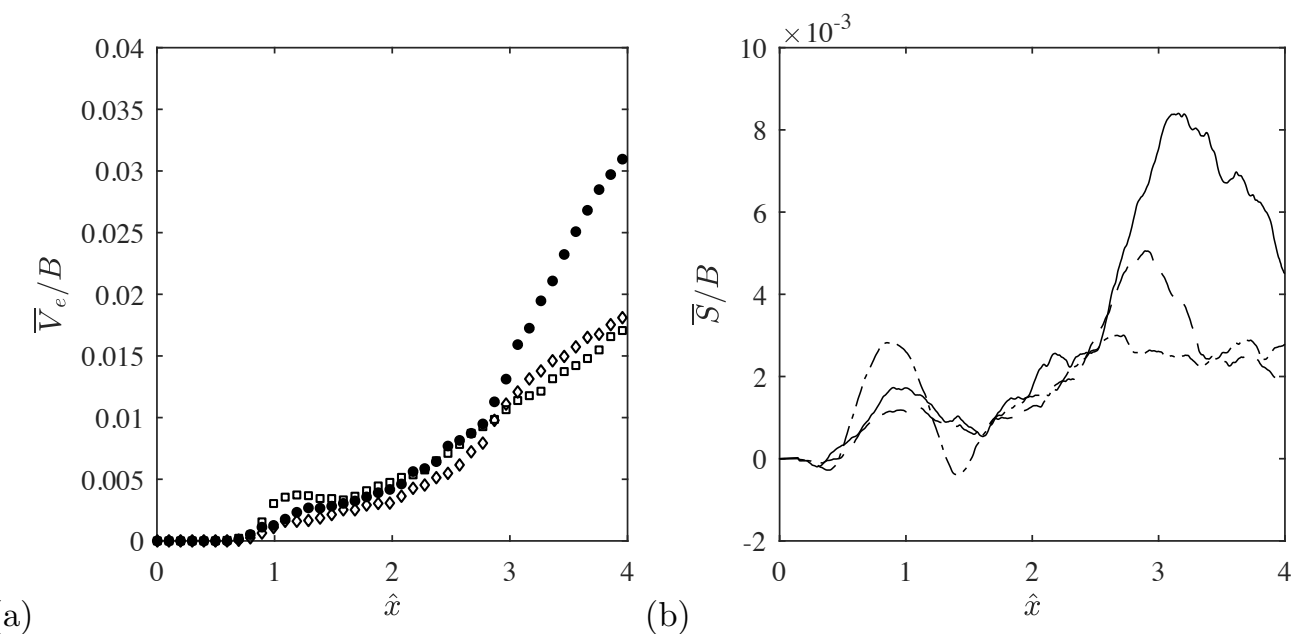

Figure 13. (a) Entrained air volume per unit half-beam along the wake $\overline{V_{e}}(\hat{x}) / B$. • case A; $\diamond$ case B; and $\square$ case C. (b) Average surface entrainment rate per unit half-beam $\overline{\mathcal{S}}(\hat{x}) / B$ along the wake. Wake distance scaled with ship speed $\hat{x}=x / F r$. — case $\mathrm{A} ;---$ case $\mathrm{B}$; and - - case C.

$\mathrm{C}$ entrain about the same amount, which is consistent with the maximum average void fraction in the DW.

We derive the surface entrainment rate $\mathcal{S}$ by considering mass conservation in an infinitesimal control volume at $x$ of size $-\infty<y<\infty ;-\infty<z<z_{i}$, where $z_{i}$ is the vertical location of the interface. Let $w(x, y, t)$ be the entrainment rate of the dimensionless $v_{e}(\vec{x}, t)$ through the interface with units of velocity $[L / T]$. Mass conservation for the location $x$ obtains

$$
\int_{-\infty}^{\infty} d y w v_{e}\left(x, y, z_{i}\right)=\int_{-\infty}^{\infty} d y \int_{-\infty}^{z_{i}} d z \nabla_{x y} \cdot\left(\vec{u}_{x y} v_{e}\right)+\frac{d}{d t} \int_{-\infty}^{z_{i}} d z v_{e}(x, y, z, t),
$$

where $\vec{u}_{x y}(x, y, z, t)$ is the $x-y$ planar velocity components $(u, v)$ and $\nabla_{x y}$ represents the planar gradient. Knowing there is no net flux in the $y$-direction, the rate of total entrainment $\mathcal{S}$ between $x$ and $x+\delta x$ is then (with units $\left[L^{2} / T\right]$ )

$$
\mathcal{S}(x, t)=\int_{-\infty}^{\infty} d y w v_{e}\left(x, y, z_{i}\right)=\int_{-\infty}^{\infty} d y \int_{-\infty}^{z_{i}} d z \frac{\partial}{\partial x}\left(u v_{e}\right)+\frac{d}{d t} \int_{-\infty}^{z_{i}} d z v_{e}(x, y, z, t) .
$$

Assuming that $u \approx U$ and taking a time average, the average surface entrainment rate is

$$
\overline{\mathcal{S}}(x)=U \frac{d}{d x} \int_{-\infty}^{\infty} d y \int_{-\infty}^{\infty} d z \overline{v_{e}}(x, y, z)=U \frac{d}{d x} \overline{V_{e}}(x) .
$$

Figure $13 \mathrm{~b}$ shows the average surface entrainment rate $\overline{\mathcal{S}}(\hat{x})$ per unit half-beam $B$ using the derivative of a local polynomial fit of $\overline{V_{e}}$. There is an initial peak average surface entrainment rate near $\hat{x} \approx 1=\hat{x}^{*}$ and a secondary peak average surface entrainment rate in the far wake $\hat{x}=\hat{x}^{d}$. Table 3 contains these locations and surface entrainment rates. For the initial peak, $\hat{x}^{*} \approx \hat{x}_{c}$ for case $\mathrm{A}$, which is expected as the average interface isosurface (see figure 6 ) shows the CCW colliding on the centerline intact. For cases B and $\mathrm{C}, \hat{x}^{*} \sim 0.9$, implying that the beam geometry is not a factor in the location of the peak entrainment. The small amount of wave breaking for $x<x_{c}$ in case B shifts $\hat{x}^{*}$ towards that of case $\mathrm{C}$ rather than case $\mathrm{A}$. The value of $\overline{\mathcal{S}}\left(\hat{x}^{*}\right) / B$ for case $\mathrm{C}$ is approximately 
twice that of case A due to the wave breaking in the CCW region. The scaling of $\hat{x}^{*}$ with the absence of the beam geometry shows that the gravity-driven ballistic scaling that held surprisingly well for the wake interface geometry characteristics is not necessarily an entrainment scaling. The secondary peak in the average entrainment rate that occurs in the DW region has an inverse dependence on $B$. This is also the same with $\overline{\mathcal{S}}\left(\hat{x}^{d}\right) / B$. We discuss this point further in $§ 5.2$.

\subsection{Air entrainment size spectrum}

We calculate the average bubble-size density spectra $\bar{N}\left(r_{\text {eff }} ; b\right)$ utilizing the individual cavity volume $v_{e}^{i}$ and effective spherical radius $r_{\text {eff }}^{i}$ (see appendix A for definitions and details). Figure 14 shows the average spectra computed in the sub-domains of the different regions in the wake (here $\forall$ in (A 2) is each sub-domain volume). In the CCW region, $x<x_{c}$ (figure $14 \mathrm{a}$ ), the cavities have relatively small effective radii. The entrainment in the breaking $\mathrm{CCW}$ of case $\mathrm{C}$ generates significantly more cavities than cases $\mathrm{A}$ and $\mathrm{B}$ due to the fully overturning CCW. In the RT region or for our purposes $x_{c}<x<x_{B}$ (figure14b), all three cases have very similar air cavity distributions. In the DW region or $x>x_{B}$ (figure 14c), the three cases have similar distributions for $r_{\text {eff }}<0.1$. However, case A has significantly more cavities for $r_{\text {eff }}>0.1$, which indicates why case A has the maximum average void fraction, average entrainment volume and subsequently largest average entrainment rate. The maximum cavity radius present and the total number of cavities increases from the CCW to DW region.

To understand the dependence of the spectrum in $y$, we separate the DW region (figure 14c) into two subregions. Figure $14 \mathrm{~d}$ shows the air cavity density spectra for the central core of the DW region, or $|y| \leqslant B$. This spectrum is near identical in magnitude and slope to the RT region in figure $14 \mathrm{~b}$. Figure $14 \mathrm{e}$ shows the DW for $|y| \geqslant B$ where the generation of larger bubbles due to the wave overturning is clear. For these two subregions, the volume in the density spectra is the DW subdomain region of figure $14 \mathrm{c}$ for comparison. Note that for $r_{\text {eff }}<0.1$, the two subregions contribute almost equally to the total spectrum in figure $14 \mathrm{c}$.

Of interest to the modeling community is the distribution of the bubble-size spectra, or specifically the slope $\beta$ of the power-law $N(r) \sim r^{\beta}$. Table 4 lists the power-law exponent corresponding to figure 14(a-c). The power-law exponent for $r_{\text {eff }}<0.1$ ranges from $-5<\beta<-4$ as long as significant air is being entrained (i.e. not for cases $\mathrm{A}$ and $\mathrm{B}$ in the $\mathrm{CCW}$ region). The power-law exponent for cases $\mathrm{A}$ and $\mathrm{B}$ in the $\mathrm{CCW}$ region is effectively the same; however, case B has a slightly smaller slope and a larger number of cavities than case A, which is consistent with the observation that there is a small amount of overturning in this region for this case. Additionally, the power-law exponents for case $\mathrm{C}$ in the breaking $\mathrm{CCW}$ and DW region are effectively the same. Finally, there is a slight increase in $\beta$ with $B / D$ in the RT and DW regions.

The value of $\beta$ depends on many physical processes. Dimensional analysis for the spectrum above the Hinze scale obtains a value of $-10 / 3$ for a given entrainment rate (Garrett et al. 2000). Experiments of unsteady breaking waves support this value (Deane \& Stokes 2002). In appendix B, we show through energy conservation that the initial breakup of a cavity by a turbulent field in the absence of any other physical mechanism produces this same $-10 / 3$ value. This value however is only valid for the initial moment of a cavity breakup. Further breakup by turbulence and buoyancy effects will increase the number of smaller bubbles and decrease the number of larger bubbles (Deane \& Stokes 2002; Garrett et al. 2000). As the values in table 4 represent long time averages of entrainment, turbulent breakup and buoyancy, a value $\beta>-10 / 3$ is expected. The slopes 

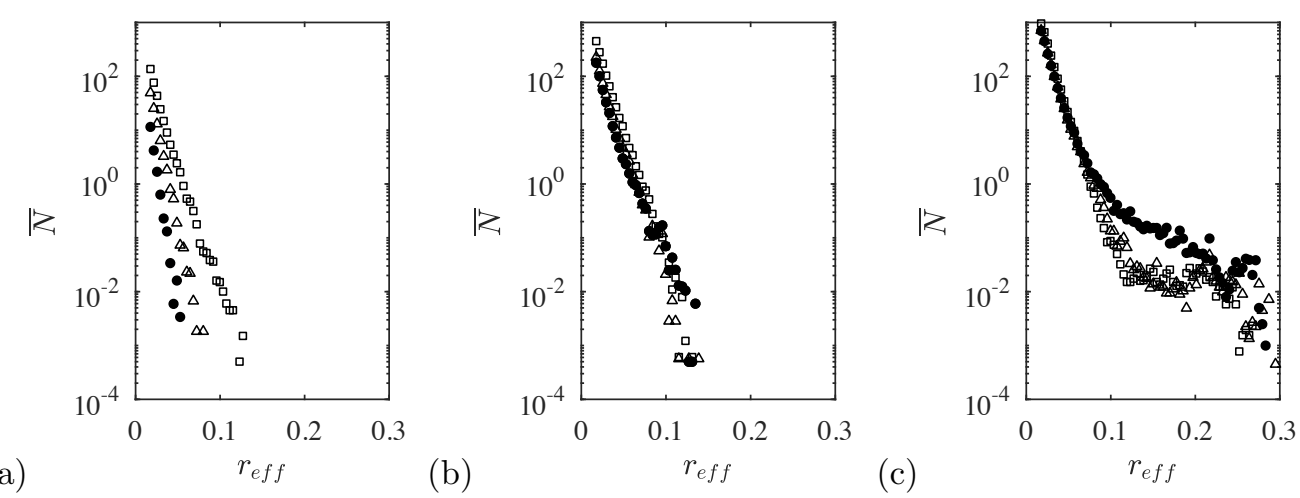

(b)
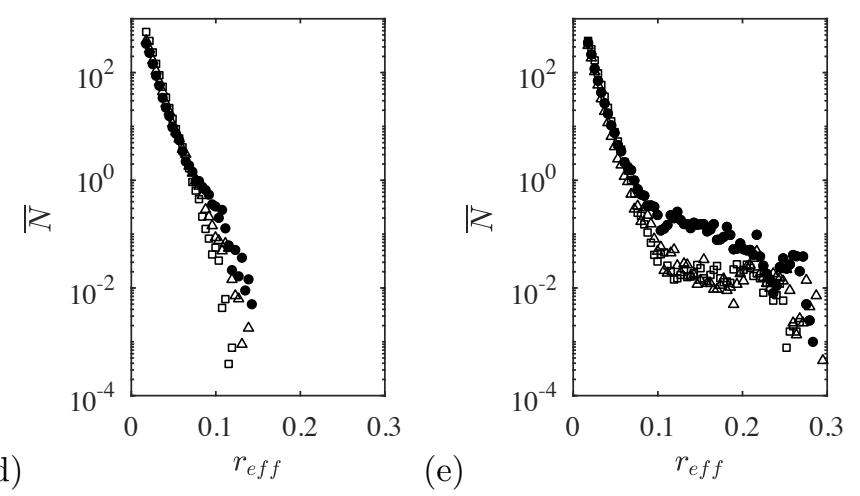

FiguRE 14. Average cavity density spectra as a function of effective radius $\bar{N}\left(r_{\text {eff }}\right)$ for all three cases: $\bullet$ case A; $\triangle$ case B; and $\square$ case C. (a) CCW $x<x_{c}$; (b) RT $x_{c}<x<x_{B}$; (c) DW for $x>x_{B}$; (d) DW for $|y| \leqslant B$; and (e) DW $|y| \geqslant B . x_{B}$ defined in table 2. Data normalized by each measurement volume $\forall_{i}$ and cavities with $r_{\text {eff }}<a_{N}$ not shown.

$$
\begin{array}{cccc}
\text { Case } & \text { CCW } & \text { RT } & \text { DW } \\
\text { A } & -7.71 & -4.38 & -4.25 \\
\text { B } & -7.19 & -4.42 & -4.48 \\
\text { C } & -4.92 & -4.52 & -4.82 \\
\text { Mean } & - & -4.44 & -4.51
\end{array}
$$

TABLE 4. Power-law exponent $\beta$ for $\bar{N} \sim r_{\text {eff }}^{\beta}$ for $r_{\text {eff }}<0.1$ in the CCW, RT and DW region.

in table 4 are consistent with the experiments of a turbulent entraining ship hull boundary layer $(\beta=-4.36)$ where multiple physical effects, including entrainment, buoyancy, and turbulence, are present (Masnadi et al. 2018).

\section{Summary}

We present simulations and analysis of the three-dimensional air entraining flow in the wake of a canonical surface ship for a range of half-beam-to-draft ratios $0.75 \leqslant$ $B / D \leqslant 2.1$ and dry draft-based Froude numbers $2.38 \leqslant F r \leqslant 3$, matching the values in laboratory scale experiments (Drazen et al. 2010). The high-resolution iLES employ stateof-the-art conservative Volume-of-Fluid (Weymouth \& Yue 2010) and Boundary Data 
Immersion methods (Weymouth \& Yue 2011) to enable robust and accurate prediction of the large-scale flow field and air entrainment for the complex air entraining flow. The analysis of air entrainment utilizes a newly developed Lagrangian method, borrowed from image processing techniques and modified to ensure conservation of bubble volume in the post processing phase, to efficiently identify regions of connected air cavities to isolate entrainment. We observe reasonably good agreement between simulations and related experimental data.

The analyses provide new insight into the flow structure and large-scale air entrainment characteristics within the near wake of a canonical surface ship. Analysis of the mean interface characteristics provide a detailed description of the three regions of the near wake: the converging corner wave $(\mathrm{CCW})$, rooster tail (RT) and diverging wave (DW). It confirms that the geometric wake characteristics in the CCW region scale ballistically, similar to the behavior of supercritical chute piers. A small parametric study of the rectangular geometry and stern speed in appendix $\mathrm{C}$ confirms that the convergent corner waves are ballistic (gravity driven). This scaling obtains a collapse with draft Froude number and geometry for $x_{c}$, the location at which the CCW collide on the centerline, as $x_{c}\left(\sqrt{2} \cos \phi_{c}\right)=B F r$ and that the convergent wave angle $\tan \theta_{c}=\left(\sqrt{2} \cos \phi_{c}\right) / F r$. The angle $\phi_{c}=45^{\circ}$ is consistent with theory.

Examination of the mean flow structure in the mixed region immediately downstream of the three-dimensional geometry has only one main similarity to that of two-dimensional hydraulic jumps (for the geometries considered): the presence of a shear layer at the transition between the bulk water and mixed region. In the very near wake, there is no appreciable evidence of a recirculating region situated above a dividing streamline, making the mixed region flow behind the stern significantly different than two-dimensional sterns or supercritical hydraulic jumps. We identify the presence of a streamwise jet with secondary vorticity inside the mixed region above the shear layer and below the average interface that originates from the three-dimensional quasi-steady breaking wave originating from the corners of the geometry. Further downstream in the diverging wave region, the breaking waves reintroduce this three-dimensional structure that might resemble a roller-bore with a strong cross-stream advection on an oblique plane that directly influences the air entrainment (and turbulent statistics as shown in part 2) in this region.

Lagrangian analysis of the air entrainment in the wake shows that it is strongest where wave breaking occurs and the maximum void fraction and amount of entrained volume depend upon the geometry. For the cases considered here, the narrowest geometry produces the largest void fractions and total volume of entrained air due to the fact that it generates large cavities in the DW region. The distribution of the average entrained air provide estimates of the average surface entrainment rate $\overline{\mathcal{S}}(\hat{x})$. An initial peak of the surface entrainment rate occurs in the CCW region and scales with $F r$. A secondary peak of the surface entrainment rate occurs near the beginning of the DW where the diverging waves break and entrain additional air cavities. The location of the secondary peak and its associated peak value have a weak and inverse dependence on $B$. The average air cavity density spectra obtained from the Lagrangian cavity volume show that the powerlaw exponent for an effective spherical radius $r_{\text {eff }}<0.1$ ranges from $-5<\beta<-4$ under active air entrainment. There is a slight increase in $\beta$ with $B / D$ in both the RT and DW regions.

Within realistic constraints on computational resources which limit the present simulations to moderate (effective) Reynolds numbers and cavity sizes large relative to physical Hinze scales, this work establishes new understanding for the complex flow and air entrainment in the near wake of a transom stern. Within these limitations, we are 
able to obtain physical insights and quantification of the scaling and characteristics of the wake geometry, flow structure and large-scale air entrainment. Part 2 of this paper (Hendrickson \& Yue submitted) adopts an Eulerian analysis framework that combines all of the surface fluctuations, spray and entrainment into a single, mixed-phase region and presents the analysis and modeling of this incompressible highly-variable density turbulent flow.

\section{Acknowledgements}

This work was funded by the Office of Naval Research N00014-10-1-0630 under the guidance of Dr. Ki-Han Kim and Dr. Thomas C. Fu. The computational resources for the effort were funded through the High Performance Modernization Program at the Department of Defense. The authors are indebted to Dr. L. Patrick Purtell at the Office of Naval Research for years of previous support that developed the capabilities for this project.

\section{Supplementary Material}

We have included six movies for upload corresponding with figures 3 , 4, and 5 .

\section{Appendix A. Lagrangian data labeling}

This section describes a Lagrangian technique for distinguishing when a volume fraction $0<f<1$ identifies a cell that is part of the bulk fluid region or entrainment (or conversely spray). Used in computer vision techniques, the two-pass standard CCA algorithm identifies and labels regions of connected (binary) data (Samet \& Tamminen 1988), where a cell is considered connected to an adjacent cell if they both contain the binary data. The first pass temporarily assigns labels to contiguously connected threedimensional regions and the second pass reduces each label to its smallest equivalence. In CCA, it is standard practice to threshold the data prior to the first pass to permit a fine-grained analysis of the connected components and ensure separation between large and small cavities.

CCA begins by defining the atmosphere $f_{a}^{0}$ as the large volume of air in the simulation that is bounded by the upper domain boundary and the entrained air $\hat{f}_{a}$ as any volume of air that is not connected to the atmosphere. In cases where there is no defined atmosphere, $f_{a}^{0}=0$. Knowing that $f_{a}$ represents the air volume fraction the total volume of air in the domain $\Omega$

$$
\sum_{\Omega} f_{a}=1-\sum_{\Omega} f=f_{a}^{0}+\sum_{i} \hat{f}_{a i}
$$

Here, all $\hat{f}_{a i}$ are disjoint, i.e. for any $f_{a}\left(\vec{x}_{0}, t_{0}\right) \neq 0$ there is only one $\hat{f}_{a i}$ that contributes to the sum in (A 1). Applying the standard CCA practice of thresholding to $f_{a}$ would violate (A 1) and result in a set of cavities sensitive to the thresholding levels. Thus, our modification to the standard CCA procedure occurs in the application of thresholding. The initial sweep at the largest threshold level finds and labels significant cavities of air with the two-pass CCA. Subsequent sweeps reduce the threshold level and either adds any new non-zero elements to an existing cavity (if it is so connected) or starts a new cavity. A final threshold value of zero ensures that (A 1) holds. In practice, this produces cavities insensitive to the specific threshold levels chosen.

We note here that a single threshold level of 0 is equivalent to the algorithm utilized in 


\begin{tabular}{|c|c|c|c|c|c|c|}
\hline Grid & $k \Delta$ & $\begin{array}{c}\text { Single-ph } \\
t=9\end{array}$ & $\begin{array}{c}\text { ase } \nu_{e} \Delta^{-1} \\
t=20\end{array}$ & $\delta \forall_{b}(t=20)$ & $\begin{array}{l}\text { Two-phase } \\
\nu_{e} \Delta^{-1}(\mathrm{t}=9)\end{array}$ & $\beta$ \\
\hline $64^{3}$ & 0.0491 & $1.58 \mathrm{E}-2$ & $9.99 \mathrm{E}-3$ & - & - & - \\
\hline $128^{3}$ & 0.0245 & $1.73 \mathrm{E}-2$ & $7.57 \mathrm{E}-3$ & $-8.0 \mathrm{E}-13$ & $3.64 \mathrm{E}-2$ & $-3.4 \pm 0.7$ \\
\hline $256^{3}$ & 0.0123 & $1.66 \mathrm{E}-2$ & $6.38 \mathrm{E}-3$ & $-5.2 \mathrm{E}-14$ & $3.37 \mathrm{E}-2$ & $-3.5 \pm 0.3$ \\
\hline $512^{3}$ & 0.00613 & $1.43 \mathrm{E}-2$ & $5.22 \mathrm{E}-3$ & $-3.0 \mathrm{E}-15$ & $2.93 \mathrm{E}-2$ & $-3.7 \pm 0.4$ \\
\hline $1024^{3}$ & 0.00307 & $1.23 \mathrm{E}-2$ & $4.41 \mathrm{E}-3$ & $-3.1 \mathrm{E}-17$ & $2.50 \mathrm{E}-2$ & $-3.8 \pm 0.2$ \\
\hline
\end{tabular}

TABLE 5. Details for two sets of Taylor-Green vortex simulations using iLES. Single-phase simulations $\nu_{e} \Delta^{-1}$ at $t=9$ and $t=20$. Two-phase air bubble simulations normalized fraction of bubble volume lost $\delta \forall_{b}=\left(1-\forall_{b}(t=20) / \forall_{b}(0)\right) / N_{t}$ over $N_{t}$ time steps, $\nu_{e} \Delta^{-1}$ at $t=9$, and initial spectrum slope $\beta$.

Deike et al. (2016). Testing of the single and multilevel threshold algorithm showed near identical performance. However, the single threshold level incorrectly groups bubbles with $r \sim \Delta$ that are a distance slightly larger than $\Delta$ from a large bubble with that larger bubble, biasing the volume of the larger cavity. The multilevel threshold algorithm correctly identifies both bubble volumes as if they were on the grid individually.

The information from the modified CCA algorithm provides calculation of the volume of the individual air cavity $v_{e}^{i}=\int \hat{f}_{a i}(\vec{x}) d \vec{x}$ and effective spherical radius $r_{\text {eff }}^{i}=\left[\frac{3}{4 \pi} v_{e}^{i}\right]^{1 / 3}$ in appendix B.2 and $\S 5.1$. To determine the average air cavity density spectra $\overline{N\left(r_{\text {eff }} ; b\right)}$ within a given liquid fluid volume $\forall$, we first count the number of cavities $n\left(r_{\text {eff }}\right)$ within each effective radius bin $b$ and define $N\left(r_{\text {eff }} ; b\right)=n\left(r_{\text {eff }}\right) /(\forall b)$. A time average is performed such that the average air cavity density spectra is

$$
\overline{N\left(r_{\text {eff }} ; b\right)}=\frac{1}{T} \int_{t}^{t+T} \frac{n\left(r_{\text {eff }}, t ; b\right)}{\forall b} \mathrm{~d} t .
$$

The units of the air cavity density spectra are $L^{-3} \mathrm{~d} r^{-1}$ (Deane \& Stokes 2002).

\section{Appendix B. Verification of the Simulation Method}

We perform verification of the Navier-Stokes flow solver and the cVOF method for single- and two-phase flows using the complex strain field provided by an evolving TaylorGreen vortex (TGV) (Taylor \& Green 1937). In previous work, TGV simulations are used to provide characterization of iLES (Drikakis et al. 2007; Zhou et al. 2014), and to study the interaction of vortices and microbubbles (Druzhinin \& Elghobashi 1998; Ferrante \& Elghobashi 2007). We use TGV simulations to quantify, for both single- and twophase flows, the effective viscosity of our iLES as a function of grid size and confirm the expected peak dissipation rate at time $t^{*} \approx 9$, a rate of decay of kinetic energy $\kappa$ of $t^{-1.2}$ for $t>t^{*}$, and its transition to $t^{-2}$ at large time (Drikakis et al. 2007). For the two-phase simulations, we verify the volume conservation of cVOF and confirm its ability to capture small-scale entrainment by turbulence.

For our simulations, we use triply-periodic cubic domain of length $2 \pi$ with initial velocity field: $\overrightarrow{\mathbf{u}}_{0}=u_{0}[\cos (k x) \sin (k y) \cos (k z),-\sin (k x) \cos (k y) \cos (k z), 0]$. We employ symmetry planes to decrease the domain size by a factor of 8 without loss of generality (Aspden et al. 2008). For two-phase simulations, we introduce a single air bubble $\left(\rho_{\text {air }} / \rho_{\text {water }}=0.001\right)$ of diameter $k d=0.5$ located at $(x, y, z)=(0.58,1.55,1.81)$. Table 5 contains the simulation details. The physical Reynolds number is $R e=u_{0} k / \nu$. 
(a)

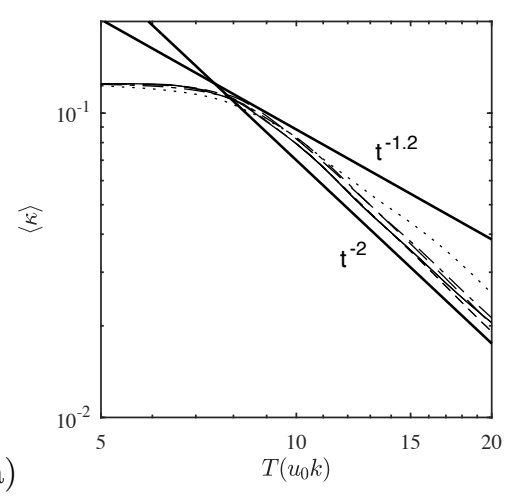

(c)

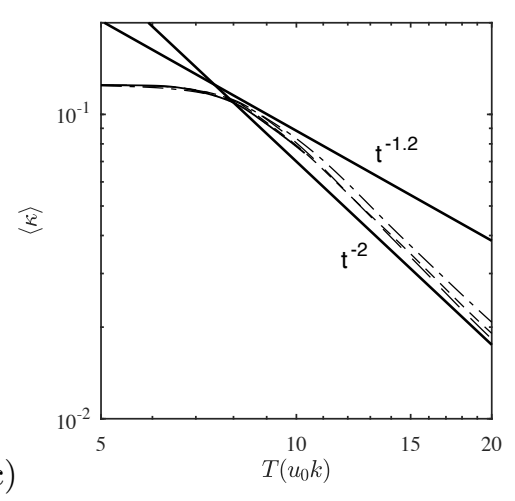

(b)

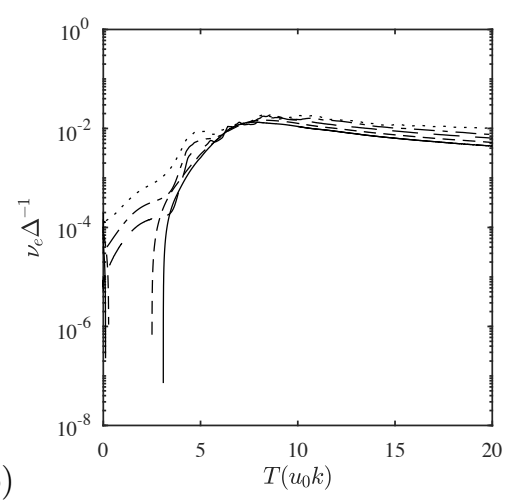

(d)

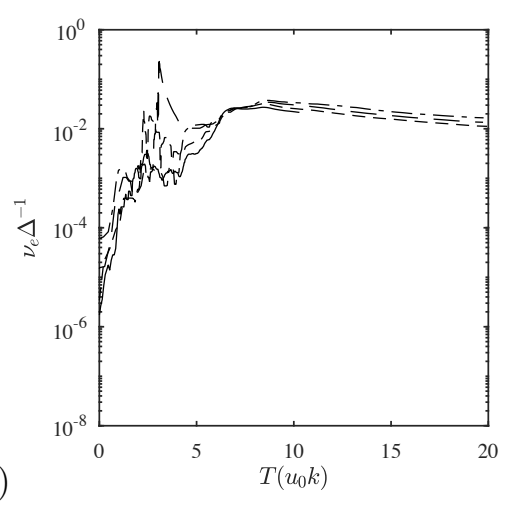

Figure 15. (a,c) Evolution of kinetic energy $\langle\kappa\rangle$ and (b,d) grid-scaled effective viscosity $\nu_{e} \Delta^{-1}$ as a function of time for an inviscid simulation of Taylor-Green vortex on varying grids. $1024^{3} ;---, 512^{3} ;---, 256^{3} ;---, 128^{3} ; \cdots, 64^{3}$; and in (a) $\_, t^{-1.2}$ and $t^{-2}$ power laws. (a-b) Single-phase simulations. (c-d) Two-phase simulations.

\section{B.1. Single-phase TGV simulations}

Figure 15a shows the evolution of $\langle\kappa\rangle$ in the entire volume as a function of time, for the range of resolutions $k \Delta$ considered. The energy decays as $t^{-1.2}$ for $t>t^{*} \approx 9$, and $t^{-2}$ in the later stages $(t \gtrsim 11)$, as expected. We estimate the iLES effective Reynolds number (or equivalently viscosity $\nu_{e}$ ) by comparing the instantaneous energy dissipation rate $\langle\epsilon\rangle=d\langle\kappa\rangle / d t$ to the square of the strain tensor $\mathcal{D} \equiv 2\left\langle s_{i j} s_{i j}\right\rangle$, where $\kappa=1 / 2 \rho u_{i}^{2}$, $s_{i j}=1 / 2\left(u_{i, j}+u_{j, i}\right),\langle\rangle$ represents the volume average and ()$_{, i}=\partial() / \partial x_{i}$ (Aspden et al. 2008). The iLES effective numerical viscosity is then

$$
\nu_{e}=\frac{\langle\epsilon\rangle}{2\left\langle s_{i j} s_{i j}\right\rangle},
$$

which requires instantaneous volumetric measurements of $d\langle\kappa\rangle / d t$ and $\mathcal{D}$ to statistically determine $\nu_{e}$ for each simulation. Figure $15 \mathrm{~b}$ shows effective viscosity $\nu_{e} \Delta^{-1}$ within the volume using (B 1), showing that $\nu_{e}$ scales approximately linearly with $\Delta$. The energy dissipation rate reaches a peak value at $t=T\left(u_{0} k\right) \approx 9$. Table 5 shows the peak value of the grid-scaled effective viscosity $\nu_{e} \Delta^{-1}$ at $t=9$ and during the decay at $t=20$. 


\section{B.2. Two-phase TGV Simulations}

In the two-phase TGV evolution, our main interest is the initial $\left(t<t^{*}\right)$ phase where air from the single large bubble entrains into the water, resulting in bubbles covering a range of smaller sizes (once entrained, the entrained bubbles themselves fragment resulting in even smaller bubbles and as the evolution continues, $t>O(10)$, the result is a large bubble cloud). We establish the mass conservation of the cVOF solver by calculating the normalized fraction of bubble volume from the cVOF function $f$ as $\forall_{b} \equiv \sum(1-f) / N_{x y z}$, summed over all points $N_{x y z}=N_{x} N_{y} N_{z}$ in the computational domain. The normalized fraction of bubble volume lost is effectively within machine accuracy throughout the simulation $(t \gtrsim 20)$ for all grids considered, as shown in table 5 . We verify the two-phase iLES dissipation characteristics in the same manner as the single-phase simulations. Figure 15(c-d) shows the evolution of $\langle\kappa\rangle$ and scaled effective viscosity $\nu_{e} \Delta^{-1}$ in the entire volume as a function of time for a range of $\Delta$. The decay of the kinetic energy follows the $t^{-1.2}$ and $t^{-2}$ power laws and the effective viscosity continues to show scaling with grid at the peak vortex decay $(t=9)$ in the same manner as the single-phase simulations (e.g figure $15 \mathrm{~b})$. The estimated values of the grid-scale effective viscosity at the peak $\left(t^{*} \approx 9\right)$ are in table 5 and have the dependence of $\nu_{e}$ on $\Delta$ given by $\nu_{e} \approx 0.036 \Delta$.

To quantify the expected initial power-law slope of $N(r) \sim r^{\beta}$ (see appendix A for definition of $N$ ) of the small bubbles that entrain from the larger bubble, we compare it to what can be theoretically expected from an energy argument. Consider the initial breakup of a large cavity into a spectrum of smaller bubbles in a turbulent flow. Assuming absence of surface tension and gravity, the dominant external force on the bubbles is the gradient of (large-scale) dynamic pressure (Hinze 1955), $\Pi=\nabla P$, where $P$ is the pressure varying over the scale of the large cavity. For a bubble of size $r$, the force acting on it is therefore $\sim \Pi r^{3}$. Using an entrainment analogy (Brocchini \& Peregrine 2001), the entrainment distance from the large cavity would scale as $d_{r} \sim r$, and hence the work done scales as $W \sim \Pi r^{4}$. The total energy required to entrain all cavities of given bubble size $r$ in volume $\forall$ is therefore $E_{r} \sim \Pi r^{4} \cdot N(r) d r \cdot \forall$. We now consider the turbulent kinetic energy at scale $r \sim k^{-1}$ given by $\kappa_{r} \sim E(k) d k \sim r^{-2} E\left(r^{-1}\right) d r$ per volume, where $E(k)$ is the turbulence power spectrum. The amount of energy for entrainment comes from turbulence in the volume surrounding the initial large cavity (with surface area $A$ times the entrainment distance $d_{r}$ ). Therefore, the total available turbulent kinetic energy in the entrainment volume for bubble size $r$ is $\kappa_{r} A d_{r} \sim A r^{-1} E\left(r^{-1}\right) d r$. Equating this to total energy required for the cavity breakup $E_{r}$, we finally obtain $N(r) \sim r^{-5} E\left(r^{-1}\right)$. For large Reynolds number flows and small bubble size $r$ (compared to the large cavity), we argue that $E(k)$ can be described by a Kolmogorov energy spectrum, $E(k) \sim k^{-5 / 3}$. This obtains the power-law bubble size spectrum $N(r) \sim r^{-10 / 3}$ for the initial breakup of a large cavity into smaller bubbles.

Figure 16 plots the average bubble-size density spectra $\bar{N}\left(r_{\text {eff }}\right)$ obtained from iLES (from the initial $t<t^{*}$ entrainment phase) with different grid resolutions $\Delta$, where $\forall$ in (A 2) is the computational domain, the time average is over one vortex period $\left(u_{o} k\right)^{-1}$. Note that the original large bubble is not included. At these effective Reynolds numbers, we expect that the variation of iLES effective viscosity in table 5 should have minimal effect on large-scale statistics. From figure 16, the largest entrained bubbles are however

$r_{\text {eff }} k \lesssim O\left(10^{-1}\right)$. The results show reasonable collapse with $\bar{N}\left(r_{\text {eff }}\right)$ close to the $r_{\text {eff }}^{-10 / 3}$ power law within the error of the fit. The theoretical value of $-10 / 3$ we derive is for the expected size distribution of air entrainment across a (single, large) interface, which does not account for effects such as fragmentation of the bubbles after they are entrained. For cVOF, we are able to quantify the bubbles in the bulk with time, but not directly the air 


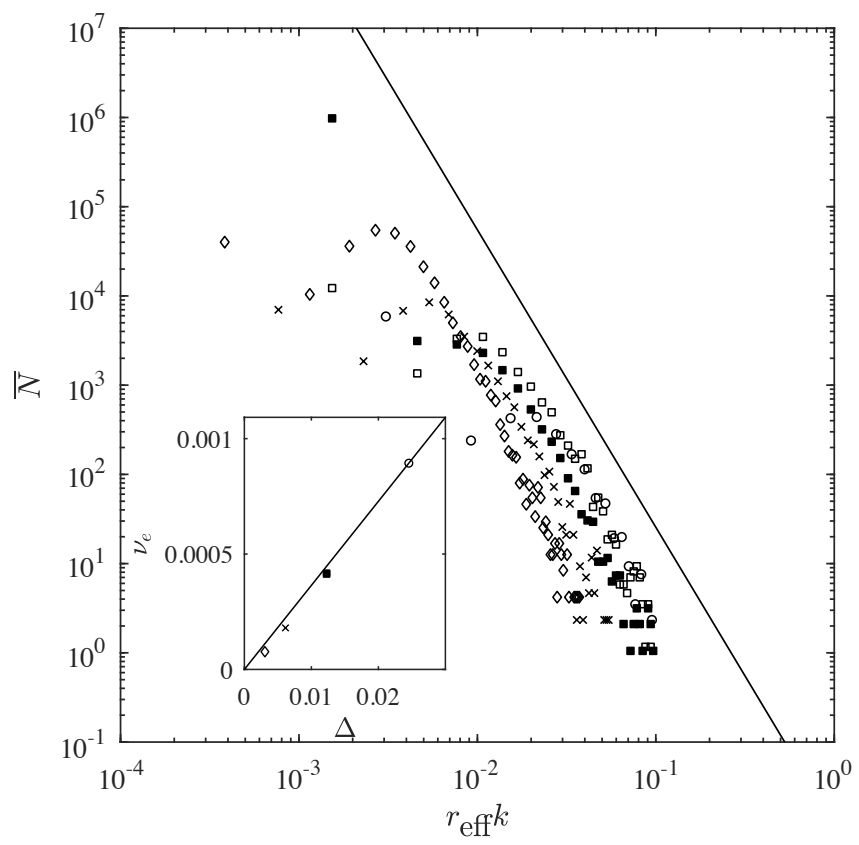

Figure 16. Two-phase Taylor Green vortex: bubble-size density spectra $\bar{N}$ of the initial entrainment of bubbles $r k \ll 1$ from a large bubble plotted as a function of effective spherical radius normalized by vortex wavenumber $r_{\text {eff }} k$. iLES with $\circ 128^{3} ; \square 256^{3} ; \times 512^{3} ; \diamond 1024^{3}$ grids; and $256^{3}$ with turbulent Weber $=1000 .-r^{-10 / 3}$. Inset effective viscosity $\nu_{e}$ as a function of grid $\Delta$ for the same symbols and $-\nu_{e}=0.036 \Delta$.

entrainment which is inferred from the bulk statistics. Thus it is, in general, difficult to separate the entrainment from the fragmentation effects in the bubble size spectra. For these results (averaged over period $T$ within the initial phase of entrainment), bubble fragmentation within the averaging window plays a role, and $\beta=-10 / 3$ is expected to be an upper bound, as the bulk bubble spectral slope is known to evolve and generally decrease with time (Deane \& Stokes 2002; Deike et al. 2016).

As $k \Delta$ decreases (from 0.05 to 0.003 , table 5 ), the $r_{\text {eff }}^{-10 / 3}$ power law range extends

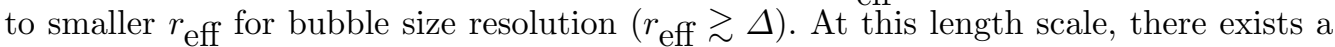
numerically-induced length scale due to the volume-conservative VOF reconstruction bias towards bubbles with sizes larger than the grid size $\Delta$. Intuitively, in any conservative VOF scheme, cavities with radius $a<\Delta$ and spacing $\sim \Delta$ will be reconstructed as a single air volume. We identify $a_{N} \approx \Delta$ as the numerical Hinze scale (Weymouth et al. 2010) with bubble-size distribution for $a<a_{N}$ suppressed ( $a_{N}$ acting as a numerical surface tension). We consider bubbles larger than $a_{N} \approx \Delta$ resolved and well represented. (Note that spectra presented in $§ 5.2$ do not include bubbles for $a<a_{N}$.) Owing to the differences in effective viscosity and influence of fragmentation, the convergence of $\bar{N}\left(r_{\text {eff }}\right)$ with $k \Delta$ within the resolved $r_{\text {eff }} k$ region can be resonably discerned.

Finally, to show that the bubble distributions in the resolved scales are unaffected by (weak) surface tension, we include a two-phase TGV simulation using a turbulent Weber number $W e=\rho_{\text {water }} \overline{u^{\prime 2}} d / \sigma=1000$. We choose this value using the magnitude of the RMS velocity fluctuations presented in part 2 (Hendrickson \& Yue submitted), the 


$\begin{array}{lccccc}\text { Case } & F r & B / D & \tan \phi_{c} & \frac{\tan \theta_{c} F r}{\alpha} & \frac{x_{c} \alpha}{F r B} \\ \text { HS } & 2.38 & 0.75 & 1.36 & 1.19 & 0.93 \\ \text { ES } & 2.38 & 1.0 & 1.24 & 1.03 & 1.03 \\ \text { GS } & 2.38 & 1.25 & 1.20 & 1.00 & 1.07 \\ \text { A } & 2.53 & 1.0 & 0.99 & 1.01 & 1.07 \\ \text { D } & 2.53 & 1.0 & 1.00 & 1.20 & 0.98 \\ \text { B } & 2.53 & 1.25 & 0.99 & 1.01 & 1.10 \\ \text { C } & 2.53 & 1.77 & 1.17 & 1.03 & 0.92 \\ \text { DS } & 2.53 & 1.0 & 1.32 & 1.05 & 1.01 \\ \text { FS } & 3.0 & 1.0 & 1.21 & 1.00 & 1.06\end{array}$

TABle 6. Cases used in parameterization study. The grid resolution is a constant $\Delta=D / 64$ in the interrogation domain. The computational parameters for $[\mathrm{D}-\mathrm{H}] \mathrm{S}$ cases are $L_{x} \times L_{y} \times L_{z}=10 D \times 6 D \times 3 D, \Delta t=0.005$ and $\delta t=0.1$. Case D is a full-geometry case with $L_{x} \times L_{y} \times L_{z}=13 D \times 10 D \times 3 D, \Delta t=0.001$ and $\delta t=0.05$.

size of the air cavities reported in $\S 5$, and the physical scale of the problem (see $\S 3$ ). We include the surface tension force in the governing equations using a continuum force and standard height-function method (Popinet 2009). From figure 16, we see that the presence of surface tension has a relatively small effect on the (resolved portion of the) bubble spectrum, with $\bar{N}$ for $W e=1000$ slightly decreased especially for smaller $r_{\text {eff }}$, as expected.

\section{Appendix C. Scaling of 3D dry transom wakes}

The results of $\S 4.2$ reveal the underlying scaling of the wake characteristics with the ship wake geometric and air entrainment characteristics. This appendix explores the scaling of these characteristics by considering additional speeds $F r=(2.38,3.0)$ and geometries $B / D=(0.75,2.1)$. For numerical expediency, the cases included in this section employ the use of a symmetry plane boundary condition on the centerline of the stern $y=0$. The details of the cases considered for this analysis are in table 6 . The inclusion of the symmetry plane enables a smaller time step and thus increases the iLES effective viscosity by an order of magnitude.

First, we perform an analysis and comparison to understand the implications of the use on the symmetry plane in the simulations. Mathematically, the symmetry plane condition requires the statistics of $u_{i}^{\prime}$ to be unchanged about that plane and that $v=\bar{v}+v^{\prime}=0$ on the plane $y=0$. These two facts imply: (i) $\overline{u^{\prime} v^{\prime}}=0$ and $\overline{v^{\prime} w^{\prime}}=0$ on the $y=0$ plane; (ii) $\overline{u^{\prime} v^{\prime}}$ and $\overline{v^{\prime} w^{\prime}}$ are symmetric about this plane; and (iii) $v^{\prime} \sim 0$ at $y=0$. Based on this, we conclude that for features where the turbulence statistics are most relevant, we do not expect the results from cases utilizing the symmetry plane assumption to be accurate. Extensive analysis obtains the mean velocity and interface for cases with the symmetry plane are quantitative and qualitatively similar when compared to results without the symmentric center plane. Quantities involving turbulent fluctuations and stresses (and as a result the air entrainment) have appreciable quantitative (and some qualitative) differences. This is sufficient for us to use this data to further elucidate the scaling arguments presented in $\S 4.2$.

The ballistic scaling arguments for the CCW flow obtain expressions for the collision point $x_{c}$ and convergent corner wave angle $\theta_{c}$ (4.1). In $\S 4.2$, we established gravity as the main mechanism driving the wake characteristics immediately aft of a dry transom stern for constant Froude number. Table 6 confirms that the potential energy jump at 


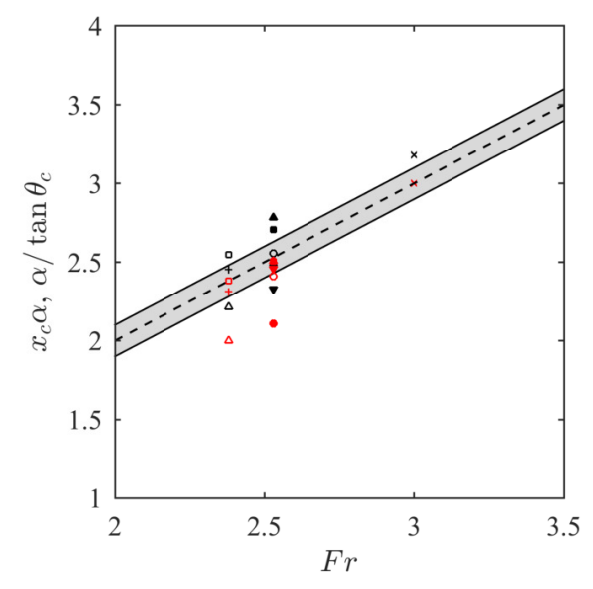

Figure 17. Scaling data from table 6. Black symbols: $x_{c} \alpha$, red symbols: $\alpha / \tan \theta_{c}$. $\mathbf{A}$; $\boldsymbol{\Delta}$ B;

$\nabla \mathrm{C} ; \bullet \mathrm{D} ; \circ \mathrm{DS} ;+\mathrm{ES} ; \times \mathrm{FS} ; \square \mathrm{GS} ; \triangle \mathrm{HS} ;$ - - - scaling from $\S 4.2$ (grey area is $\pm 10 \%$ ).

the surface $D$ is the driving mechanism for different Froude numbers and the expressions for $x_{c}$ and $\theta_{c}$ in (4.1) hold as the values are again near unity. Figure 17 is a graphical representation of the scaling for each parameter (on the line being that the scaled value is 1.0). Note in table 6 that $\phi_{c}$ is not $45^{\circ}$ for cases using the symmetry plane. They adopt a consistent value of $50^{\circ} \leqslant \phi_{c} \leqslant 53^{\circ}$, which is likely due to an interaction between the inlet condition and symmetry plane. Despite this difference, the scaling for $x_{c} \alpha / F r B$ and $\tan \theta_{c} F r / \alpha$ is still $\approx 1$, which supports the conclusion of gravity being the driving mechanism in this region.

\section{REFERENCES}

Adams, P., George, K., Stephens, M., Brucker, K. A., O’Shea, T. T. \& Dommermuth, D. G. 2010 A numerical simulation of a plunging breaking wave. Phys. Fluids 22 (9), 091111.

Aspden, A., Nikiforkakis, N., Dalziel, S. \& Bell, J. B. 2008 Analysis of implicit LES methods. Comm. App. Math. and Comp. Sci. 3 (1), $103-126$.

BALDY, S. 1993 A generation-dispersion model of ambient and transient bubbles in the close vicinity of breaking waves. J. Geophys. Res. 98 (C10), 18277-18293.

Brocchini, M. \& Peregrine, D. H. 2001 The dynamics of strong turbulence at free surfaces. Part 1. Description. J. Fluid Mech. 449, 225-254.

Chachereau, Y. \& Chanson, H. 2011 Bubbly flow measurements in hydraulic jumps with small inflow Froude numbers. Int. J. of Multiphas. Flow 37 (6), $555-564$.

Chanson, H. 1995 Air entrainment in two-dimensional turbulent shear flows with partially developed inflow conditions. Int. J. of Multiphas. Flow 21 (6), $1107-1121$.

Chanson, H. 2009 Current knowledge in hydraulic jumps and related phenomena. A survey of experimental results. Eur. J. Mech. B Fluids 28 (2), $191-210$.

Chanson, H., Aoki, S. \& Hoque, A. 2004 Physical modelling and similitude of air bubble entrainment at vertical circular plunging jets. Chem. Eng. Sci. 59 (4), 747-758.

Chanson, H. \& Brattberg, T. 2000 Experimental study of the air-water shear flow in a hydraulic jump. Int. J. of Multiphas. Flow 26 (4), $583-607$.

Chanson, H. \& Manasseh, R. 2003 Air entrainment processes in a circular plunging jet: Voidfraction and acoustic measurements. J. Fluids Eng.-Trans. ASME 125 (5), 910-921.

Cummings, P. D. \& Chanson, H. 1997 Air entrainment in the developing flow region of plunging jets - Part 2: Experimental. J. Fluids Eng.-Trans. ASME 119 (3), 603-608. 
Deane, G. B. \& Stokes, M. D. 2002 Scale dependence of bubble creation mechanisms in breaking waves. Nature 418 (6900), 839-844.

Deike, L., Melville, W. K. \& Popinet, S. 2016 Air entrainment and bubble statistics in breaking waves. J. Fluid Mech. 801, 91-129.

Dong, R. R., Katz, J. \& HuAng, T. T. 1997 On the structure of bow waves on a ship model. J. Fluid Mech. 346, 77-115.

Drazen, D. A., Fullerton, A. M., Fu, T. C., Beale, K. L.C., O’Shea, T. T., Brucker, K. A., Dommermuth, D. G., Wyatt, D. C., Bhushan, S., Carrica, P. M. \& Stern, F. 2010 A comparison of model-scale experimental measurements and computational predictions for a large transom-stern wave. In Proceedings of the 28th Symposium on Naval Ship Hydrodynamics.

Drikakis, D., Fureby, C., Grinstein, F. F. \& Youngs, D. 2007 Simulation of transition and turbulence decay in the Taylor-Green vortex. J. Turbul. 8 (20).

Druzhinin, O. A. \& Elghobashi, S. 1998 Direct numerical simulations of bubble-laden turbulent flows using the two-fluid formulation. Phys. Fluids 10 (3), 685-697.

Ferrante, A. \& Elghobashi, S. E. 2007 On the effects of microbubbles on Taylor-Green vortex flow. J. Fluid Mech. 572, 145-177.

Fu, T. C., Fullerton, A. M., Drazen, D. A., Minnick, L., Walker, D., Ratcliffe, T., Russell, L. \& Capitain, M. 2010a A detailed study of transom breaking waves. Part 2. Tech. Rep. NSWCCD-50-TR-2010/003. Naval Surface Warfare Center Carderock Div, Bethesda, MD.

Fu, T. C., Fullerton, A. M., Terrill, E. J. \& LadA, G. 2006 Measurements of the wave fields around the R/V Athena I. In Proceedings of the 26th Symposium on Naval Ship Hydrodynamics.

Fu, T. C., Ratcliffe, T., O’Shea, T. T., Brucker, K. A., Graham, R. S., Wyatt, D. C. \& Dommermuth, D. G. $2010 b$ A comparison of experimental measurements and computational predicitions of a Deep-V planing hull. In 28th Symposium on Naval Hydrodynamics. Pasadena, California.

Fullerton, A. M., Fu, T. C., Brewton, S., Brucker, K. A., O’Shea, T. T. \& Dommermuth, D. G. 2010 A comparison of measured and predicted wave-impact pressures from breaking and non-breaking waves. In 28th Symposium on Naval Hydrodynamics.

Garrett, C., Li, M. \& Farmer, D. 2000 The connection between bubble size spectra and energy dissipation rates in the upper ocean. J. Phys. Oceanogr. 30, $2163-2171$.

Hendrickson, K, Weymouth, G D, Banerjee, S \& Yue, D K.-P. 2012 Air entrainment and multiphase turbulence in the bubbly wake of a transom stern. In Proceedings of the 29th Symposium on Naval Ship Hydrodynamics.

Hendrickson, K. \& Yue, D. K.-P. submitted Wake behind a three-dimensional dry transom stern. Part 2: Analysis and modeling of incompressible highly-variable density turbulence.

Hinze, J. O. 1955 Fundamentals of the hydrodynamic mechanism of splitting in dispersion processes. AIChE Journal 1 (3), 289-295.

Hoque, A. \& Aoki, S. I. 2008 Air entrainment and associated energy dissipation in steady and unsteady plunging jets at free surface. Appl. Ocean Res. 30 (1), 37-45.

Hoyt, J. \& Sellin, R. 1989 Hydraulic jump as "mixing layer". J. Hydraul. Eng. 115 (12), $1607-1614$.

Jingsen, M., Oberai, A. A., Hyman, M. C., Drew, D. A. \& JR., R. T. Lahey 2011 Twofluid modeling of bubbly flows around surface ships using a phenomenological subgrid air entrainment model. Comput. Fluids 52, $50-57$.

Lamarre, E. \& Melville, W. K. 1991 Air entrainment and dissipation in breaking waves. Nature 351, 469-472.

Lin, C., Hsieh, S.-C. Hsieh, Lin, I-J., Chang, K.-A. \& Raikar, R. V. 2012 Flow property and self-similarity in steady hydraulic jumps. Exp. Fluids 53 (5), 1591-1616.

Ma, G., Shi, F. \& Kirby, J. T. 2011 A polydisperse two-fluid model for surf zone bubble simulation. J. Geophys. Res. 116 (5).

Maki, K. J., Troesch, A. W. \& Beck, R. F. 2008 Experiments of two-dimensional transom stern flow. J. Ship Res. 52 (4), 291-300.

Martínez-Legazpi, P., Rodríguez-Rodríguez, J., Korobkin, A. \& Lasheras, J. C. 2015 
Formation of corner waves in the wake of a partially submerged bluff body. J. Fluid Mech. $\mathbf{7 7 1}, 547-563$.

Martínez-Legazpi, P., Rodríguez-Rodríguez, J., Marugán-Cruz, C. \& Lasheras, J. C. 2013 Plunging to spilling transition in corner surface waves in the wake of a partially submerged vertical plate. Exp. Fluids 54 (1), 1-11.

Masnadi, N., Erinin, M. A., Washuta, N., Nasiri, F., Balaras, E. \& Duncan, J. H. 2018 Air entrainment and surface fluctuations in a turbulent ship hull boundary layer. In Proceedings of the 32nd Symposium on Naval Ship Hydrodynamics.

Mortazavi, M., Le Chenadec, V., Moin, P. \& Mani, A. 2016 Direct numerical simulation of a turbulent hydraulic jump: turbulence statistics and air entrainment. J. Fluid Mech. 797, 60-94.

Mossa, M. \& Tolve, U. 1998 Flow visualization in bubbly two-phase hydraulic jump. J. Fluid Eng-T ASME 120, 160-165.

Ohl, C. D., Oguz, H. N. \& Prosperetti, A. 2000 Mechanism of air entrainment by a disturbed liquid jet. Phys. Fluids 12 (7), 1710-1714.

Olivieri, A., Pistani, F., Wilson, R., Campana, E. F. \& Stern, F. 2007 Scars and vortices induced by ship bow and shoulder wave breaking. J. Fluid Eng. 129 (11), 1445-1459.

Pagliara, S., Kurdistani, S. \& Roshni, T. 2011 Rooster tail wave hydraulics of chutes. $J$. Hydraul. Eng. 137 (9), 1085-1088.

Popinet, S. 2009 An accurate adaptive solver for surface-tension-driven interfacial flows. $J$. Comp. Phys. 228 (16), $5838-5866$.

Rodriguez-Rodriguez, J, Marugan-Cruz, C., Aliseda, A. \& Lasheras, J. C. 2011 Dynamics of large turbulent structures in a steady breaker. Exp. Therm. Fluid Sci. 35, $301-310$.

Rouse, H., Вhoota, B. V. \& Hsu, E.-Y. 1949 Design of channel expansions. Proceedings of the ASCE 75 (9), 1369-1385.

Samet, H. \& Tamminen, M. 1988 Efficient component labeling of images of arbitrary dimension represented by linear bintrees. TIEEE Trans. Pattern Anal. Mach. Intell. .

TAYlor, G. I. \& Green, A. E. 1937 Mechanism of the production of small eddies from large ones. Proc. R. Soc. Lond. A Math. Phys. Sci. 158, 499-521.

Terrill, E. J. \& Fu, T. C. 2008 At-sea measurements for ship hydromechanics. In Proceedings of the 27th Symposium on Naval Ship Hydrodynamics, , vol. 1.

Terrill, E. J. \& TAYlor, G. R. L. 2015 Entrainment of air at the transoms of full-scale surface ships. J. Ship Res. 59 (1), 49-65.

Weymouth, G. D., Hendrickson, K., Banerjee, S. \& Yue, D. K.-P. 2010 Bubble source modeling using macro-scale two-phase flow simulations. In Proceedings of the 28th Symposium on Naval Ship Hydrodynamics.

Weymouth, G. D. \& Triantafyllou, M. S. 2013 Ultra-fast escape of a deformable jetpropelled body. J. Fluid Mech. 721, 367-385.

Weymouth, G. D. \& Yue, D. K.-P. 2010 Conservative volume-of-fluid method for free-surface simulations on cartesian-grids. J. Comp. Phys. 229 (8), $2853-2865$.

Weymouth, G. D. \& Yue, D. K.-P. 2011 Boundary data immersion method for Cartesian-grid simulations of fluid-body interaction problems. J. Comp. Phys. 230 (16), 6233 - 6247.

Wyatt, D. C., Fu, T. C., Taylor, G. L., Terrill, E. J., Xing, T., Bhushan, S., O'Shea, T. T. \& Dommermuth, D. G. 2008 A comparison of full-scale experimental measurements and computational predictions of the transom-stern wave of the $\mathrm{R} / \mathrm{V}$ Athena I. In Proceedings of the 27th Symposium on Naval Ship Hydrodynamics.

Zhou, Y, Grinstein, F. F., Wachtor, A. J. \& Haines, B. M. 2014 Estimating the effective Reynolds number in implicit large-eddy simulation. Phys. Rev. E 89, 013303.

Zhu, Y. G., Oguz, H. N. \& Prosperetti, A. 2000 On the mechanism of air entrainment by liquid jets at a free surface. J. Fluid Mech. 404, 151-177. 